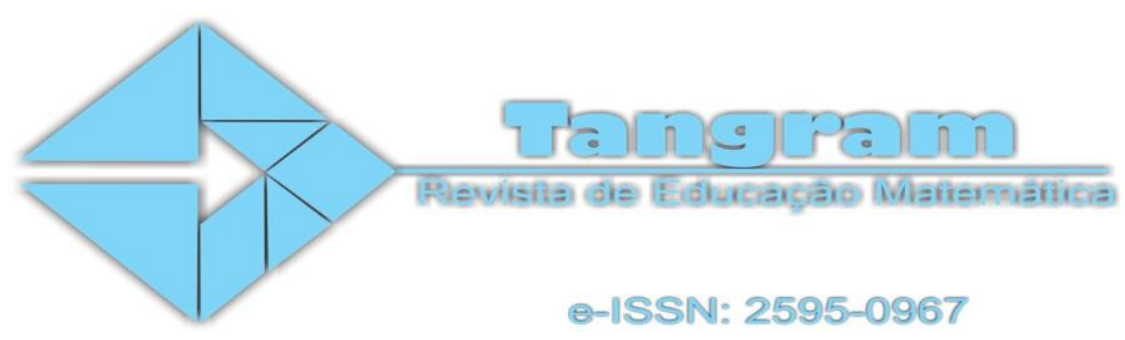

\title{
El Enfoque de los Itinerarios de Enseñanza de las Matemáticas: ¿por qué?, ¿para qué? y ¿cómo aplicarlo en el aula?
}

\section{The Mathematics Teaching Itineraries Approach: Why? What for? And how to apply it in the classroom?}

\section{A Abordagem dos Itinerários do Ensino da Matemática: por quê?, Por quê? E como aplicá-lo na sala de aula?}

\author{
Ángel Alsina \\ Universidad de Girona, Departamento de Educación \\ Girona, España \\ e-mail: angel.alsina@udg.edu \\ Orcid: 0000-0001-8506-1838
}

Enviado:24/05/2020

Aceito: $18 / 06 / 2020$

DOI: 10.30612/tangram.v3i2.12018

\begin{abstract}
Resumen: Se describe el Enfoque de los Itinerarios de Enseñanza de las Matemáticas (EIEM), que plantea la enseñanza a partir de secuencias intencionadas que consideran contextos informales (situaciones de vida cotidiana, materiales manipulativos y juegos), contextos intermedios (recursos literarios y tecnológicos) y contextos formales (recursos gráficos), y se ofrecen cinco recomendaciones para aplicar el EIEM en el aula: 1) planificar y gestionar la enseñanza de los contenidos a través de los procesos matemáticos;2) promover prácticas de enseñanza que consideren tanto al alumnado como al profesorado; 3 ) considerar contextos informales, intermedios y formales en todas las secuencias, con distinto protagonismo según el nivel escolar; 4) garantizar el principio de abstracción progresiva, desde lo concreto hacia lo abstracto; y 5) disponer de criterios objetivos para la selección de los contextos de enseñanza de las matemáticas.
\end{abstract}

Palabras clave: Enfoque de los Itinerarios de Enseñanza de las Matemáticas. Enseñanza de las matemáticas. Aprendizaje de las matemáticas. Práctica docente. Desarrollo profesional del profesorado de matemáticas.

Tangram - Revista de Educação Matemática, Dourados - MS - v.3 n.2, pp. 127-159 (2020) 


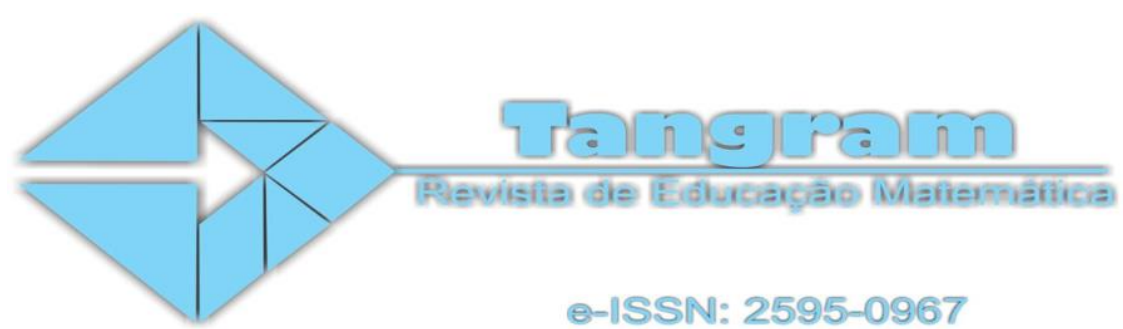

Abstract: The Mathematics Teaching Itineraries Approach (MTIA) is described, which proposes teaching based on intentional sequences that consider informal contexts (situations of daily life, manipulatives and games), intermediate contexts (literary and technological resources) and formal contexts (graphic resources), and five recommendations are offered to apply the MTIA in the classroom: 1) plan and manage the teaching of content through mathematical processes; 2) promote teaching practices that consider both the student and the teacher; 3) consider real, intermediate and formal contexts in all sequences, with a different role depending on the school level; 4) guarantee the principle of progressive abstraction, from the concrete to the abstract; and 5) have objective criteria for the selection of mathematics teaching contexts.

Keywords: Mathematics Teaching Itineraries Approach. Mathematics teaching. Mathematics learning. Teaching practice. Professional development of mathematics teachers.

Resumo: Neste artigo é descrita a Abordagem de Itinerários de Ensino de Matemática (MTIA), que propõe ensino baseado em sequiências intencionais que consideram contextos informais (situações da vida cotidiana, manipulativos e jogos), contextos intermediários (recursos literários e tecnológicos) e contextos formais (recursos gráficos), e cinco recomendações são oferecidas para aplicar o MTIA na sala de aula: 1) planejar e gerenciar o ensino de conteúdo por meio de processos matemáticos; 2) promover práticas de ensino que considerem o aluno e o professor; 3) considerar contextos reais, intermediários e formais em todas as seqüências, com um papel diferente, dependendo do nível da escola; 4) garantir o princípio da abstração progressiva, do concreto ao abstrato; e 5) possuir critérios objetivos para a seleção dos contextos de ensino de matemática.

Palavras-chave: Abordagem de Itinerários de Ensino de Matemática. Ensino de matemática. Aprendizagem de matemática. Prática de Ensino. Desenvolvimento profissional de professores de matemática.

Tangram - Revista de Educação Matemática, Dourados - MS - v.3 n.2, pp. 127-159 (2020) 


\section{Introducción}

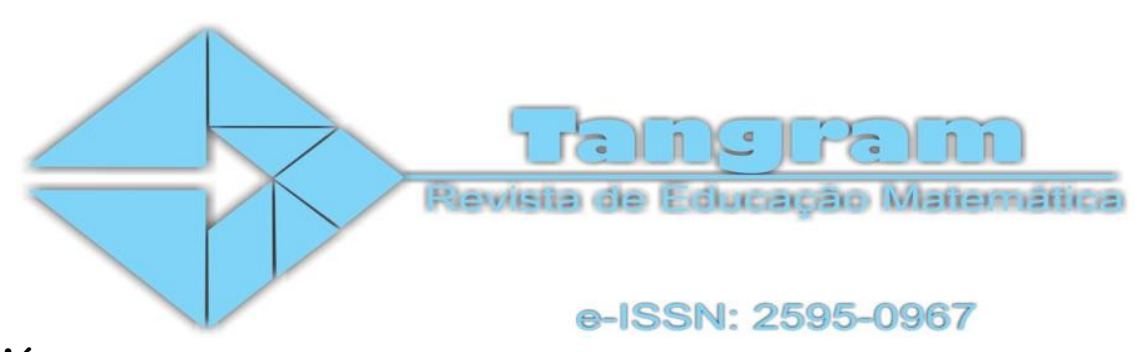

Múltiples estudios sobre la práctica de aula, el conocimiento, la competencia y el desarrollo profesional del profesorado de matemáticas realizados desde distintos modelos teóricos y en el marco de diversas agendas de investigación en educación matemática, como por ejemplo "aprender el conocimiento y destrezas útiles para enseñar matemáticas" y "planificación y gestión de la enseñanza en diversos contextos y su influencia en el desarrollo de la comprensión" (Alsina, 2019a, p. 188), revelan déficits de naturaleza muy diversa para enseñar matemáticas (Badillo, Climent, Fernández y González, 2019).

El propósito de este artículo es mucho más humilde y no pretende indagar en profundidad ni hacer un listado de las lagunas de conocimiento, pero sí que se quiere hacer una breve reflexión sobre uno de los elementos que ha generado mayores obstáculos para llevar a cabo una enseñanza eficaz: el uso (y abuso) del libro de texto como único recurso para enseñar matemáticas. Desde este prisma, se asume la idea de Stylianides (2009) cuando afirma que muchos profesores y profesoras se apoyan en los libros de texto para compensar los déficits formativos, conformándose en un recurso didáctico muy importante a la hora de interpretar el currículo y seleccionar tareas matemáticas para el alumnado. De esta forma, el libro se convierte en el recurso preponderante para organizar el proceso de enseñanza y aprendizaje (Remillard, 2000), ya que expone de manera concreta una transposición didáctica del saber (Chevallard, 1991). En otras palabras, la enorme influencia del libro de texto en las prácticas de enseñanza de las matemáticas no deja espacio para que el profesorado llegue a conocer muchas maneras de actuar y ejercitarlas en la práctica, además de disponer de criterios para saber cuándo, qué y por qué algo es conveniente y reflexionar sobre ello sistemáticamente, en el sentido expuesto desde la perspectiva del Modelo Realista de Formación del Profesorado (Korthagen, 2001).

La experiencia acumulada a lo largo de muchos años a partir de múltiples actividades de formación permanente del profesorado llevadas a cabo en toda la geografía española y en

Tangram - Revista de Educação Matemática, Dourados - MS - v.3 n.2, pp. 127-159 (2020) 


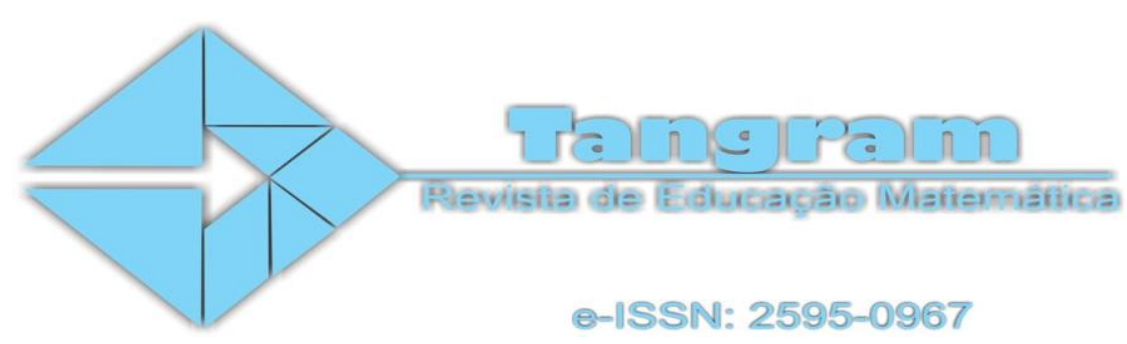

diversos países de América Latina me ha proporcionado, tal como se indica en Alsina (2019b), el enorme privilegio de que muchos maestros y maestras de las etapas de Educación Infantil y Primaria principalmente me hayan abierto las puertas de sus aula y las ventanas de sus mentes para poder ver de primera mano sus prácticas de aula y escuchar sus dudas, sus inquietudes, sus dificultades y, sobre todo, sus deseos de mejorar la enseñanza de las matemáticas para impulsar que sus alumnos y alumnas sepan usar los conocimientos matemáticos de forma comprensiva y eficaz en todas las situaciones de su vida cotidiana en las que dichos conocimientos son necesarios.

El Enfoque de los Itinerarios de Enseñanza de las Matemáticas (EIEM, a partir de ahora) pretende ofrecer una respuesta modesta a estas necesidades del profesorado, para avanzar hacia una enseñanza eficaz y hacia prácticas productivas en el aula de matemáticas. Por un lado, y considerando el Principio de Enseñanza del NCTM (2003, p. 17), "una enseñanza eficaz requiere conocer lo que el alumnado sabe y lo que necesita aprender, y luego estimularlo y ayudarlo para que lo aprenda bien". En este sentido, para ser eficaz, el profesorado debe conocer y entender profundamente las matemáticas que enseña y ser capaz de hacer uso de este conocimiento con flexibilidad. Necesita comprender a sus alumnos y alumnas y confiar en ellos como aprendices de matemáticas y como seres humanos, y ser cuidadosos al elegir y utilizar las estrategias pedagógicas y de evaluación (National Comission on Teaching and America's Future, 1996). Además, la eficacia docente requiere reflexión y esfuerzos continuos para conseguir mejorarla (Alsina y Mulà, 2019). Por otro lado, por práctica productiva en educación matemática se entiende "una acción o destreza educativa útil y provechosa para promover el aprendizaje de las matemáticas con sentido en todos los niveles escolares" (Alsina, en prensa).

Desde esta perspectiva, pues, la finalidad de este artículo consiste en describir el EIEM y proporcionar diversas recomendaciones para su aplicación en el aula, en el marco de una escuela que se renueva.

Tangram - Revista de Educação Matemática, Dourados - MS - v.3 n.2, pp. 127-159 (2020)

Este obra está licenciada com uma Licença Creative Commons Atribuição-NãoComercial-CompartilhaIgual 3.0 Brasil. 


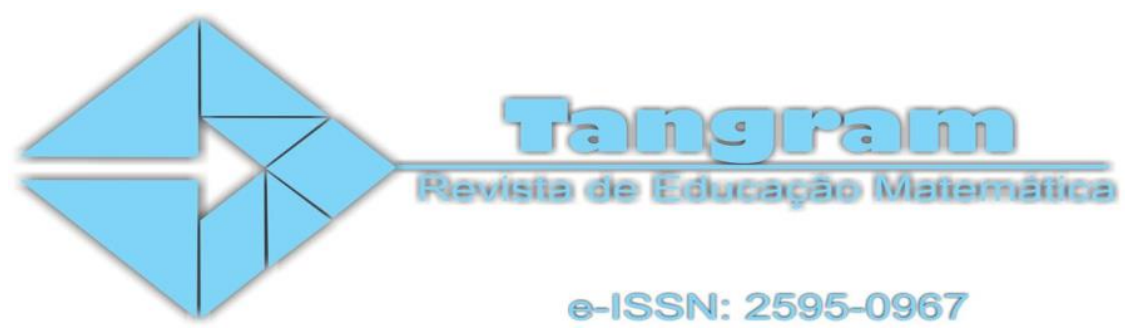

Fundamentación teórico-metodológica del Enfoque de los Itinerarios de Enseñanza de las Matemáticas.

Retomando la fundamentación inicial descrita en Alsina (2020) y, tal como se muestra en la figura 1, desde una perspectiva teórica y metodológica el EIEM se sustenta principalmente en tres pilares engranados entre ellos: la Perspectiva Sociocultural del Aprendizaje Humano (Vygostsky, 1978); el Modelo Realista de Formación del Profesorado (Melief, Tigchelaar y Korthagen, 2010; Tigchelaar, Melief, Van Rijswijk y Korthagen, 2010), adaptado a la formación del profesorado de matemáticas (Alsina, 2019c); y la Educación Matemática Realista (EMR) de Freudenthal (1991).

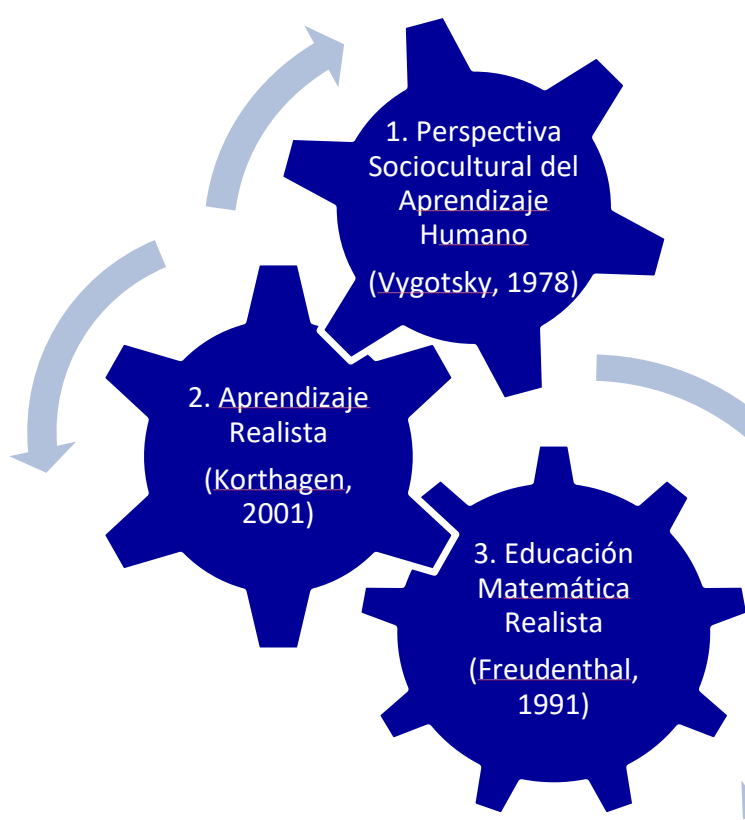

Figura 1. Fundamentos teórico-metodológicos del EIEM. Fuente: elaboración propia.

\section{La Perspectiva Sociocultural del Aprendizaje Humano.}

Tangram - Revista de Educação Matemática, Dourados - MS - v.3 n.2, pp. 127-159 (2020)

Este obra está licenciada com uma Licença Creative Commons Atribuição-NãoComercial-CompartilhaIgual 3.0 Brasil. 


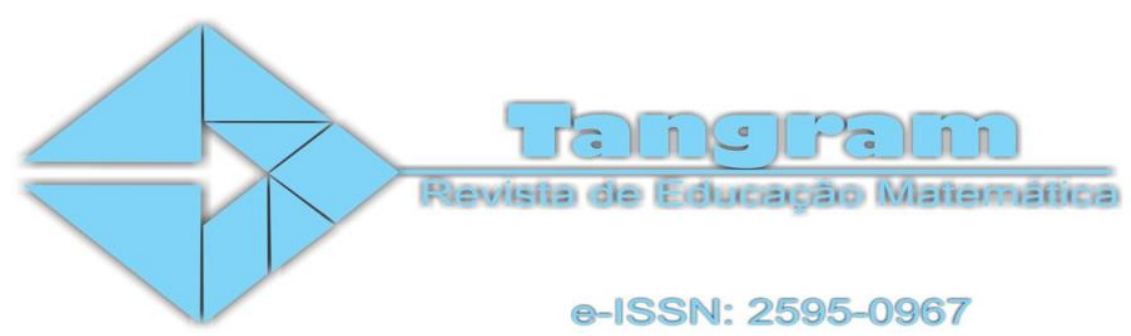

Esta perspectiva, que se fundamenta en las aportaciones de Vygotsky (1978) y en las reinterpretaciones de su obra que han hecho otros autores tanto desde el ámbito de la psicología del aprendizaje (Ivic, 1994; Wertsch, 1985, 1991) como desde el campo de la educación matemática (Lerman, 2000, 2001; Schmittau, 2003; entre otros), interesa principalmente destacar que la educación se concibe como un fenómeno social y cultural que se basa en la lenguaje y en la interacción como herramientas fundamentales para promover el desarrollo de procesos psicológicos superiores, junto con la idea de que el pensamiento intelectual depende de la construcción autorregulada del conocimiento, que va de un proceso interpsicológico a un proceso intrapsicológico a través de procesos de internalización (Alsina y Domingo, 2010).

Vygotsky explica los procesos de internalización a partir de la "Ley genética general del desarrollo cultural", que plantea que toda función psicológica aparece primero en un plano social/interpsicológico, para luego hacerlo en el plano individual/intrapsicológico. En otras palabras, se refiere a la creación de procesos psicológicos superiores en el plano interno, a partir de la internalización de procesos sociales mediados semióticamente. Esta estrecha vinculación entre los planos interno y externo puede facilitar una interpretación errónea del proceso de internalización en términos de transferencia (Wertsch, 2013), considerando que se trata de un mero traspaso de propiedades de procesos externos a un medio interno, preexistente y autónomo en su desarrollo. Por el contrario, y como indica Leóntiev (1981), el proceso de internalización no consiste en la transferencia de una actividad externa a un plano interno preexistente, un plano de la conciencia, sino que es el proceso mediante el que este plano es formado. El proceso de internalización, pues, no es una simple copia de lo externo, sino que va más allá, al hacerlo propio. Cada persona lo aprehende de una manera, lo interpreta, le da un sentido en función de sus experiencias previas, de sus necesidades y de lo que le resulta significativo y, con base a ello, construye su propia imagen, su propia re-presentación -entendida como nueva presentación en la mente- personal, creativa y re-creada nuevamente por la persona. En definitiva, se trata de

Tangram - Revista de Educação Matemática, Dourados - MS - v.3 n.2, pp. 127-159 (2020) 


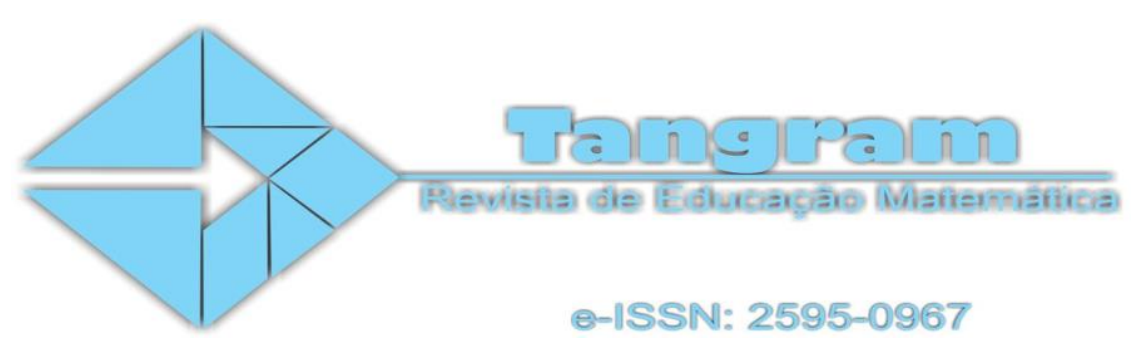

una re-elaboración con una transformación de los conocimientos, de los conceptos y de las herramientas de una sociedad determinada (Leóntiev, 1981).

Como consecuencia de lo descrito, las teorías socioculturales en educación matemática surgen de la concepción del conocimiento matemático como un proceso social y cultural (Lerman, 2000), al que hay que añadir además el planteamiento descrito sobre el proceso de internalización. Desde esta visión, el objetivo último de la educación matemática es preparar a los ciudadanos y ciudadanas para que desarrollen capacidades individuales que les permitan aprovechar al máximo las oportunidades de aprendizaje matemático provenientes de las interacciones sociales. Lerman (2001) defiende que sin las aportaciones de los demás y sin la capacidad individual de captarlas, una persona no se podría desarrollar plenamente como ser humano. Estos inputs pueden enviarse y ser captados de diferentes maneras y con el uso de diferentes lenguajes y, por lo tanto, uno de los papeles de la cultura consiste en educar y sugerir maneras diversas de percibir e interpretar la realidad (Radford, 2006, p. 108).

Schmittau (2003) hace referencia al papel del currículum de matemáticas para lograr estos propósitos. Según este autor, el currículum debería tener un alto grado de coherencia interna -entre sus elementos estructurales- pero también externa, para poder establecer vínculos con lo que la sociedad reclame y ofrezca.

\section{EI Modelo Realista de Formación del Profesorado}

De este modelo, que ha sido desarrollado en la Universidad de Utrecht (Países Bajos) por Korthagen (2001) y Melief, Tigchelaar y Korthagen (2010), entre otros, por un lado interesa destacar que pretende, a través de la reflexión sistemática, impulsar la integración de la persona con sus experiencias personales y como aprendices, con sus conocimientos teóricos y con sus representaciones sobre lo que es enseñar y aprender, razón por la cual se usa el término "realista-reflexivo" (Esteve y Alsina, 2010). Desde este prisma, como ya se ha indicado en la introducción, se asume que el profesorado debería conocer muchas

Tangram - Revista de Educação Matemática, Dourados - MS - v.3 n.2, pp. 127-159 (2020)

Este obra está licenciada com uma Licença Creative Commons Atribuição-NãoComercial-CompartilhaIgual 3.0 Brasil. 


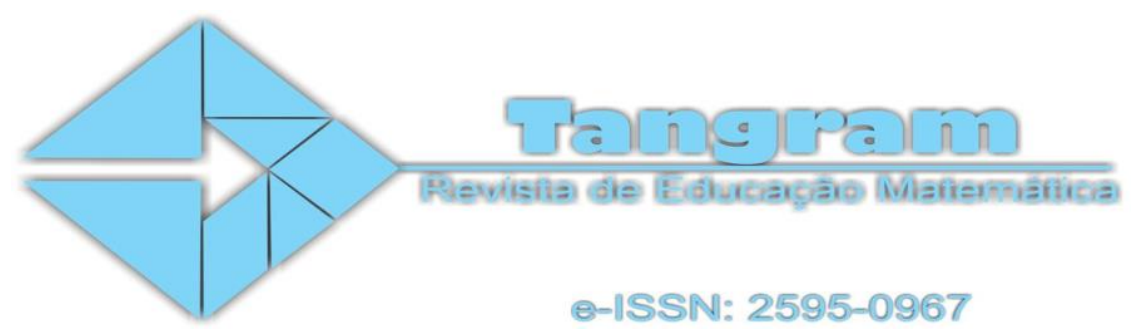

maneras de actuar y ejercitarlas en la práctica, es decir, debería disponer de criterios para saber cuándo, qué y por qué algo es conveniente y reflexionar sobre ello sistemáticamente (Korthagen, 2001). La perspectiva derivada del Modelo Realista se concreta en cinco principios sintetizados por Melief et al. (2010):

1. El punto de partida son los interrogantes que emergen de la misma práctica y que el profesorado en formación experimenta en un contexto real de aula.

2. La formación realista pretende fomentar una reflexión sistemática. El modelo ALACT que se presenta en la figura 2, que debe su nombre a las siglas en inglés Action, Looking back to the action, Awareness of essential aspects, Creating alternative methods of action y Trial, describe el proceso ideal de reflexión que se basa en una alternancia entre “acción” y "reflexión” en la que se distinguen cinco fases.

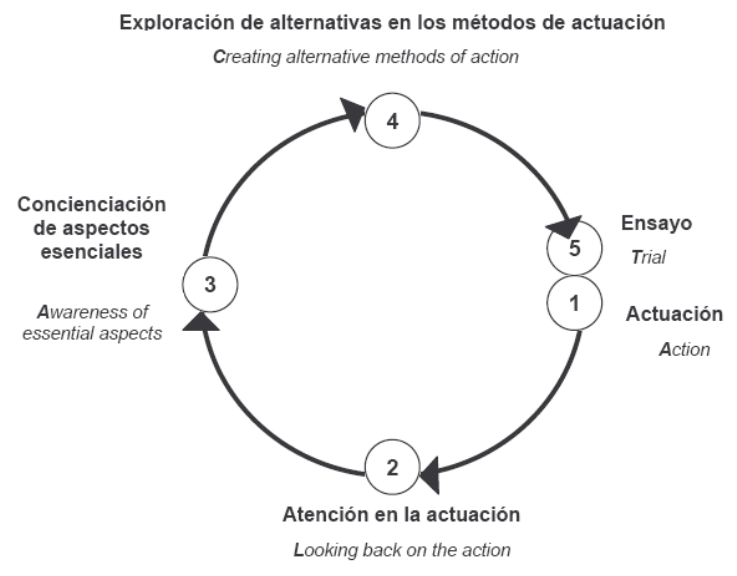

Figura 2. Modelo ALACT (Korthagen, 2001). Fuente: Alsina (2019b)

3. El aprendizaje es un proceso social e interactivo.

4. Se distinguen tres niveles en el aprendizaje: las experiencias, inquietudes y necesidades (Gestalts); los esquemas mentales (Esquemas); y las relaciones conceptuales que se establecen (Teoría).

Tangram - Revista de Educação Matemática, Dourados - MS - v.3 n.2, pp. 127-159 (2020)

Este obra está licenciada com uma Licença Creative Commons Atribuição-NãoComercial-CompartilhaIgual 3.0 Brasil. 


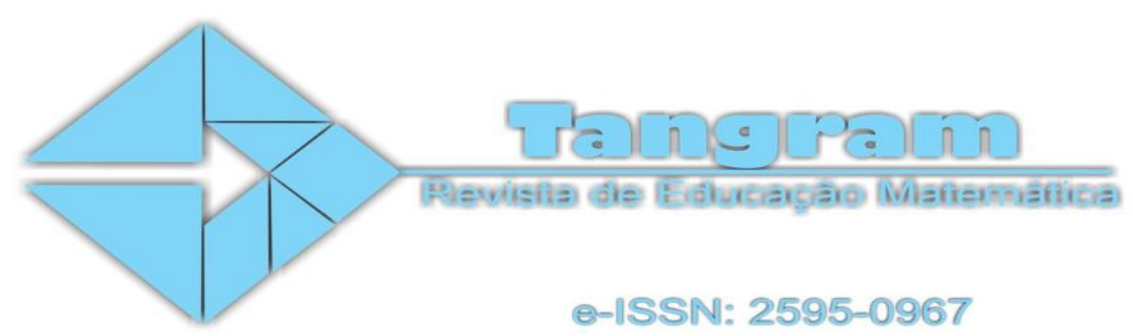

5. Se fomenta la autonomía y la construcción autorregulada del desarrollo profesional para descubrir los aspectos que se quieren o deben cambiar, además de buscar soluciones y evaluarlas.

En relación a la aplicación de este modelo en la formación de maestros de matemáticas, Alsina (2019c) presenta desde una perspectiva analítica, interpretativa y crítica los resultados de diversos estudios que muestran los avances en investigación sobre la formación de maestros de matemáticas desde el modelo realista-reflexivo. En concreto, se presentan cuatro estudios sobre diseño, aplicación y análisis de episodios desde este modelo de formación que incluyen un ciclo formativo; diversos recursos y estrategias didácticas para promover la deconstrucción de los conocimientos cotidianos (CC) del profesorado de matemáticas que pueden ser un obstáculo para su desarrollo profesional; la identificación de las marcas de autorregulación que permiten deconstruir $\mathrm{CC}$ y coconstruir y reconstruir conocimientos profesionales (CP); y, como síntesis de los estudios preliminares, la descripción del Modelo Transformacional de la Competencia Profesional del Profesorado de Matemáticas (Alsina y Mulà, 2019).

\section{La Educación Matemática Realista}

De esta teoría, que nace en el Instituto para el Desarrollo de la Educación Matemática de la Universidad de Utrecht, hoy conocido como Freudenthal Institute for Science and Mathematics Education, interesan sobre todo sus seis principios (Freudenthal, 1991), que se sintetizan y/o actualizan a continuación:

1. Principio de actividad: las matemáticas se consideran una actividad humana a la que todas las personas pueden acceder. Hacer matemáticas (matematizar) es más importante que aprenderla como producto terminado y, lo relevante, es aprender a abordar matemáticamente y críticamente los problemas que se presentan en la vida cotidiana.

Tangram - Revista de Educação Matemática, Dourados - MS - v.3 n.2, pp. 127-159 (2020)

Este obra está licenciada com uma Licença Creative Commons Atribuição-NãoComercial-CompartilhaIgual 3.0 Brasil. 


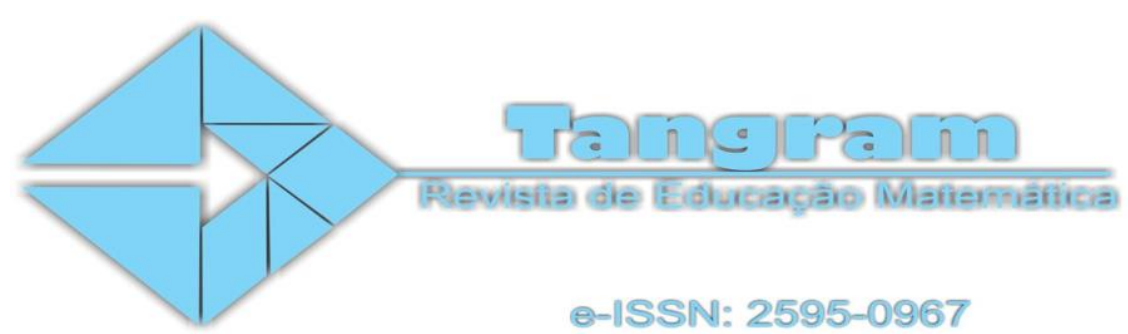

2. Principio de realidad: las matemáticas surgen como matematización (organización) de la realidad. Se aprenden haciendo matemáticas en contextos reales o realistas y, que un contexto sea o no realista, depende de la experiencia previa del alumnado y/o de su capacidad para imaginarlo o visualizarlo.

3. Principio de Reinvención Guiada: la educación matemática debe ofrecer al alumnado la oportunidad guiada de reinventar las matemáticas por parte del docente. Se reinventan modelos, conceptos, operaciones y estrategias matemáticas a través de procesos de deconstrucción, coconstrucción y reconstrucción y, para guiar este proceso, es necesaria la reflexión del profesorado acerca de los aprendizajes de su alumnado.

4. Principio de Niveles: el alumnado lleva a cabo una matematización progresiva (Treffers, 1987) a través de distintos niveles de comprensión, que incluyen el nivel situacional, en el contexto de la situación misma; el nivel referencial, que implica la esquematización a través de modelos, descripciones, conceptos y procedimientos; el nivel general, que se asocia a la exploración, reflexión y generalización; y el nivel formal, cuando se alcanzan procedimientos estándares y se usan notaciones convencionales. La matematización es horizontal cuando un problema contextual se convierte en un problema matemático, y vertical cuando, dentro de la matemática misma, se usan estrategias de reflexión, prueba, generalización, rigorización, esquematización y simbolización para avanzar en la formalización.

5. Principio de interacción: el aprendizaje de las matemáticas se considera una actividad social. Desde este prisma, la interacción entre los alumnos y las alumnas y entre el alumnado y el profesorado provoca que cada uno reflexione a partir de lo que aportan los demás, pudiendo alcanzar así niveles más altos de comprensión.

6. Principio de interconexión: la resolución de problemas reales o realistas a menudo exige establecer conexiones. Estas conexiones pueden ser intradisciplinares, lo que implica que los distintos bloques de contenido matemático (numeración y cálculo, álgebra, geometría, medida y estadística y probabilidad) no pueden ser tratados como entidades

Tangram - Revista de Educação Matemática, Dourados - MS - v.3 n.2, pp. 127-159 (2020) 


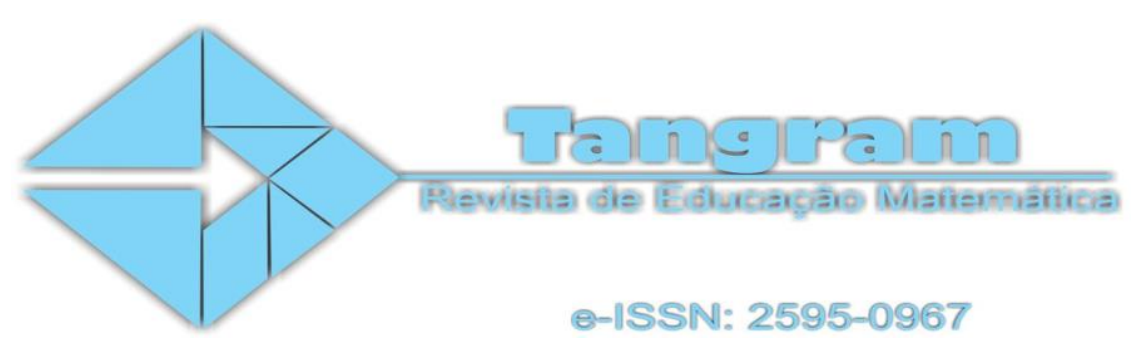

separadas; o bien interdisciplinares, entre las matemáticas y otras áreas de conocimiento como las ciencias, la tecnología, la ingeniería, las artes, etc.

En síntesis, en la EMR se utilizan situaciones de la vida cotidiana o problemas contextualizados como punto de partida para aprender matemáticas. Progresivamente, estas situaciones son matematizadas a través de modelos, mediadores entre lo abstracto y lo concreto, para formar relaciones más formales y estructuras abstractas (Heuvel-Panhuizen, 2002). Además, se apoya en la interacción en el aula (Fauzan, Plomp y Slettenhaar, 2002), junto con la idea de que al alumnado se le debería dar la oportunidad de reinventar las matemáticas bajo la guía de un docente en lugar de intentar transmitir una matemática preconstruida (De Corte, Greer y Verschaffel, 1996).

A partir de estos fundamentos teórico-metodológicos se ha diseñado el EIEM, que se describe en la próxima sección.

\section{El Enfoque de los Itinerarios de Enseñanza de las Matemáticas.}

Hace ya varios años, y con el propósito de buscar maneras ajustadas a las necesidades reales del alumnado tanto para aprender matemáticas como para aprender a usarlas de forma comprensiva y eficaz en todas las situaciones -escolares y no escolares- en las que dichos conocimientos son necesarios, Alsina (2010) planteó un diagrama piramidal en el que se comunicaba de forma sencilla y muy visual el tipo de contextos necesarios para desarrollar el pensamiento matemático y su "frecuencia de uso" más recomendable, en función de la posición que ocupa cada contexto: de más o menos frecuencia desde la base hacia la cúspide (figura 3). En este diagrama piramidal, más conocido como "Pirámide de la Educación Matemática" al hacer un símil con la pirámide de la alimentación, no se descartaba ningún recurso, sino que solo se pretendía informar sobre la conveniencia de restringir algunos de ellos a un uso ocasional y, por eso, se consideró que podía ser una herramienta útil para el profesorado preocupado por hacer de su metodología una garantía de educación matemática.

Tangram - Revista de Educação Matemática, Dourados - MS - v.3 n.2, pp. 127-159 (2020)

Este obra está licenciada com uma Licença Creative Commons Atribuição-NãoComercial-CompartilhaIgual 3.0 Brasil. 

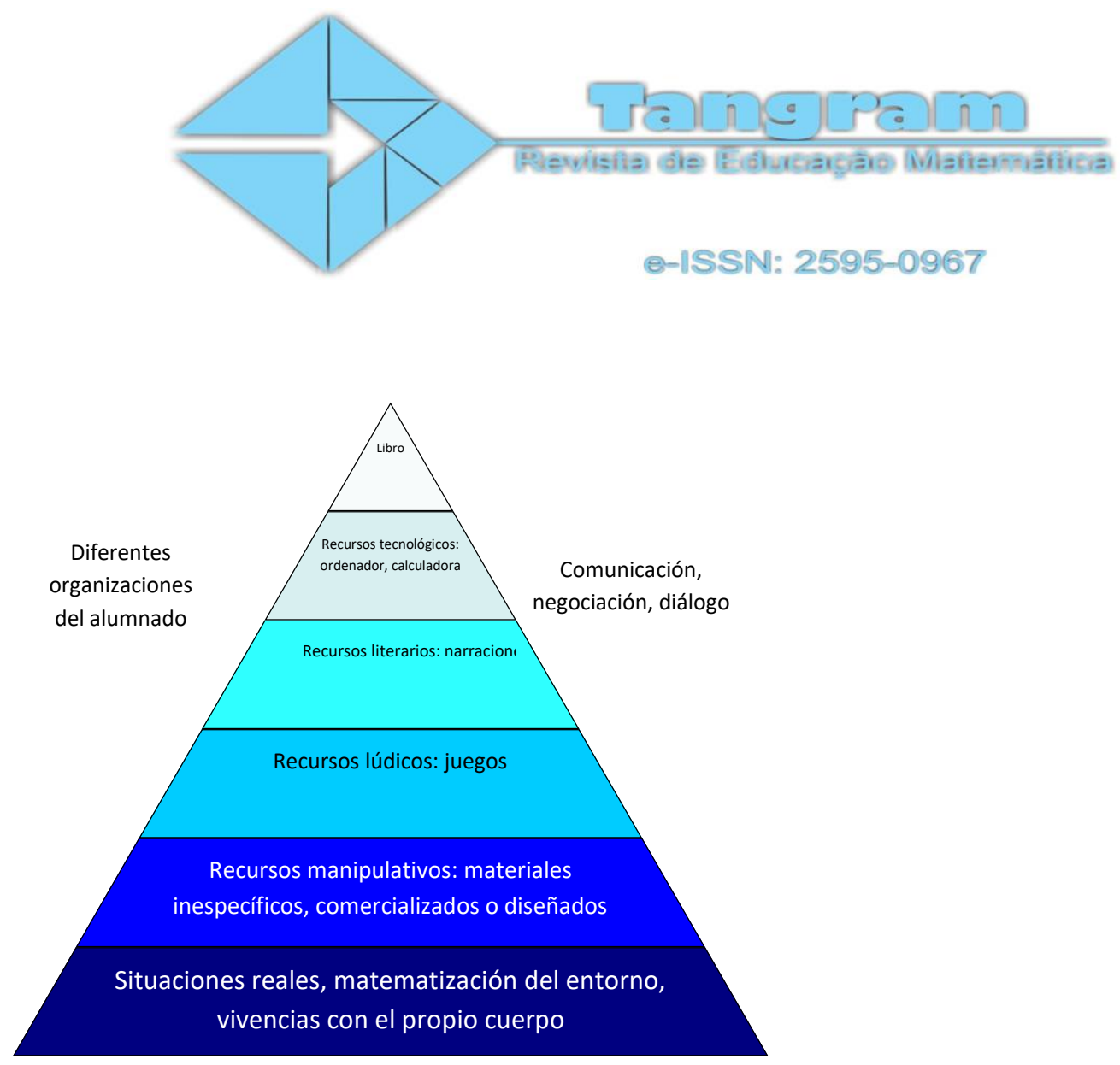

Figura 3. Pirámide de la Educación Matemática. Fuente: Alsina (2010, p. 14)

En la base se situaban los contextos que necesita todo el alumnado para aprender matemáticas y que, por lo tanto, se podrían y deberían "consumir" diariamente: las situaciones problemáticas y los retos que surgen en la vida cotidiana de cada día, la observación y el análisis de los elementos matemáticos del entorno, la manipulación con materiales diversos y los juegos, entendidos como la resolución de situaciones problemáticas. Después seguían los que deben "tomarse" alternativamente, como los recursos literarios y los recursos tecnológicos. Y, por último, en la cúspide, se ubicaban los recursos que deberían usarse de forma ocasional, concretamente los libros de texto, por las razones que ya se han expuesto en la introducción.

Tangram - Revista de Educação Matemática, Dourados - MS - v.3 n.2, pp. 127-159 (2020)

Este obra está licenciada com uma Licença Creative Commons Atribuição-NãoComercial-CompartilhaIgual 3.0 Brasil. 


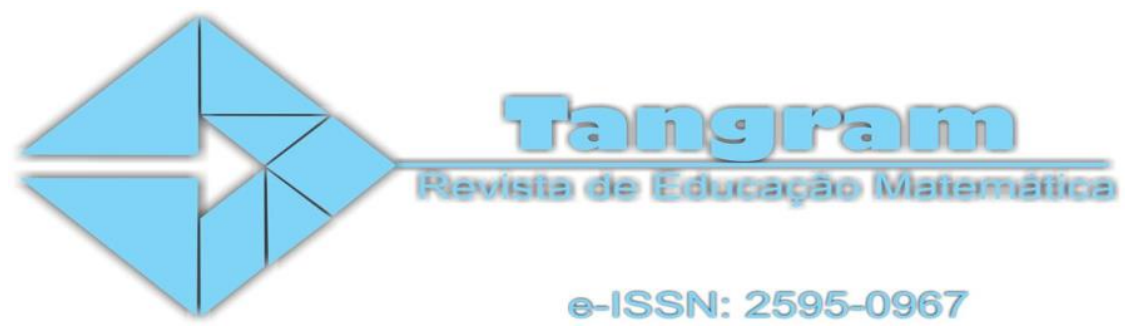

Con los años, este planteamiento ha evolucionado hacia el EIEM (Alsina, 2018, 2019b), asumiendo que la palabra "itinerario" se refiere a una secuencia de enseñanza intencionada que contempla tres niveles (figura 4):

Nivel 1

Enseñanza en contextos informales
Nivel 2

Enseñanza en contextos intermedios
Nivel 3

Enseñanza en contextos formales

Figura 4. Niveles del EIEM. Fuente: elaboración propia.

A continuación, se describen estos niveles:

1. Enseñanza en contextos informales: la enseñanza del contenido matemático se inicia en situaciones reales o realistas, como por ejemplo el entorno inmediato, o bien materiales manipulativos y juegos, en los que el conocimiento de la situación y las estrategias se utilizan en el contexto de la situación misma, apoyándose en los conocimientos informales, el sentido común y la experiencia.

2. Enseñanza en contextos intermedios: la enseñanza del contenido prosigue en contextos que hacen de puente entre los contextos reales o realistas de la fase previa y los contextos formales de la fase posterior, como por ejemplo algunos recursos literarios (cuentos y canciones) y tecnológicos (applets, robots educativos programables, etc.), que a través de la exploración y la reflexión conducen a la esquematización y generalización progresiva del conocimiento matemático.

Tangram - Revista de Educação Matemática, Dourados - MS - v.3 n.2, pp. 127-159 (2020) 


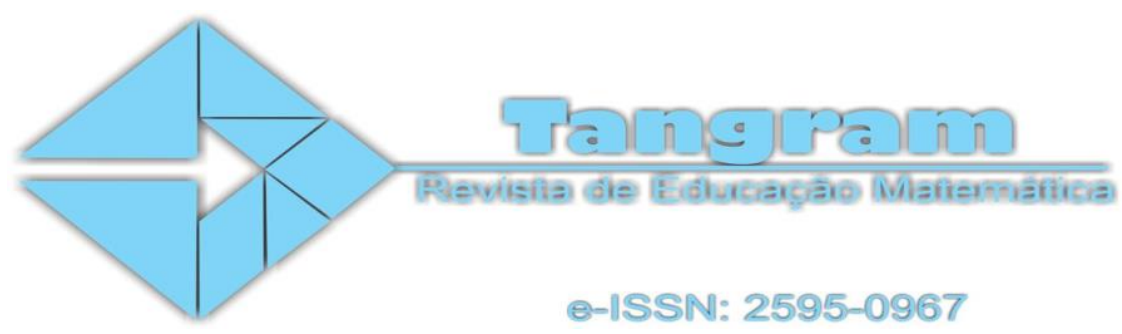

3. Enseñanza en contextos formales: la enseñanza del contenido finaliza en contextos gráficos y simbólicos, como por ejemplo las fichas y los libros de texto, en los que se trabaja la representación y formalización del conocimiento matemático con procedimientos y notaciones convencionales para completar de esta forma el aprendizaje desde lo concreto hasta lo simbólico.

Como se indica en Alsina (1029b), el EIEM se aleja de una visión de la enseñanza de las matemáticas basada en la repetición y la práctica de ejercicios que presentan los libros de texto como principales estrategias didácticas para "aprender" matemáticas, y en su lugar, plantea que es necesario fomentar la comprensión más que la mera memorización, la actividad heurística más que la pura ejercitación, o el pensamiento matemático crítico más que la simple repetición.

La comprensión, la actividad heurística y el pensamiento matemático crítico son, de hecho, algunos de los principales pilares sobre los que se sustenta la educación matemática del siglo XXI (Alsina y Planas, 2008). Necesitamos, más que nunca, formar a personas que comprendan en profundidad el conocimiento matemático para que puedan aplicarlo en todas las situaciones a lo largo de la vida en las que, de una forma u otra, están implicadas las matemáticas. Necesitamos también formar a personas que descubran por sí mismos las ideas matemáticas a través de una planificación y gestión adecuadas de las prácticas matemáticas basadas en la resolución de problemas, el razonamiento y la prueba, la comunicación, la argumentación, las conexiones, la modelización y la representación, más que transmitirles un conocimiento matemático ya construido previamente. Y, por supuesto, necesitamos formar a personas que procesen e interpreten críticamente la gran cantidad de datos que recibimos constantemente a través de diferentes medios (prensa escrita, televisión, internet, etc.). En definitiva, necesitamos formar a ciudadanos y ciudadanas que sean capaces de plantear problemas y preguntas vitales con claridad y precisión; que evalúen información relevante; que lleguen a conclusiones y soluciones, probándolas con criterios relevantes, y que se comuniquen con eficacia a la hora de idear soluciones.

Tangram - Revista de Educação Matemática, Dourados - MS - v.3 n.2, pp. 127-159 (2020)

Este obra está licenciada com uma Licença Creative Commons Atribuição-NãoComercial-CompartilhaIgual 3.0 Brasil. 


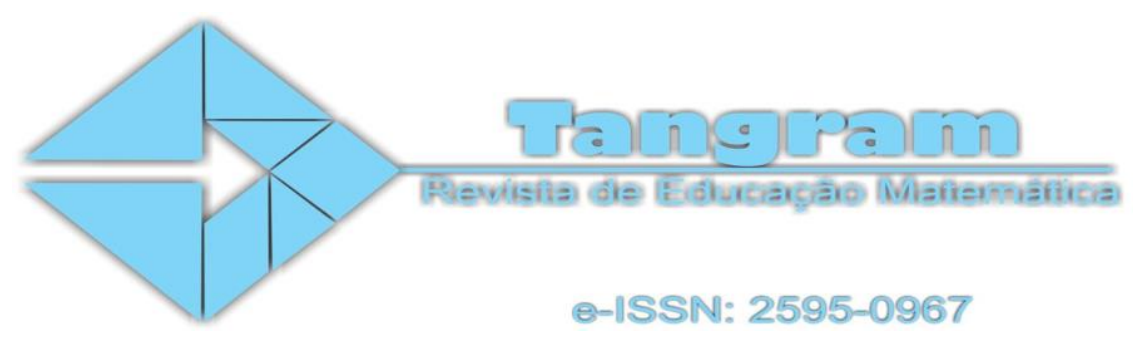

Esta visión contemporánea de la educación matemática requiere, primero, de un amplio dominio profesional de los conocimientos matemáticos a enseñar, puesto que no se puede enseñar bien lo que no se sabe y, segundo, un amplio dominio profesional acerca de las formas de enseñar dichos conocimientos, puesto que el alumnado de hoy no tiene las mismas necesidades para aprender matemáticas que el alumnado de años atrás, por lo que no tiene ningún sentido enseñar lo mismo que hace décadas y menos aún enseñarlo de la misma forma.

\section{La aplicación del EIEM en el aula: primeras recomendaciones para una enseñanza eficaz y el diseño de prácticas productivas.}

En esta última sección se proporcionan cinco recomendaciones iniciales para el profesorado interesado en aplicar el EIEM en su práctica docente. Intencionadamente, se indica que se trata de unas primeras recomendaciones puesto que, a medida que se analice la aplicación del EIEM en el aula, se irán incorporando y/o reformulando.

\section{Recomendación 1. Planificar y gestionar la enseñanza de los contenidos a través de los procesos matemáticos.}

Esta primera recomendación se fundamenta en las aportaciones del NCTM (2003) acerca del conocimiento matemático, que considera diez estándares: cinco estándares de contenidos (números y operaciones, álgebra, geometría, medida, y análisis de datos y probabilidad) y cinco estándares de procesos (resolución de problemas, razonamiento y prueba, comunicación, conexiones y representación). Este planteamiento curricular, de acuerdo con Alsina (2012), implica partir de un enfoque mucho más globalizado que no se limite a trabajar los contenidos aisladamente, sino de forma integrada. Además, exige

Tangram - Revista de Educação Matemática, Dourados - MS - v.3 n.2, pp. 127-159 (2020) 


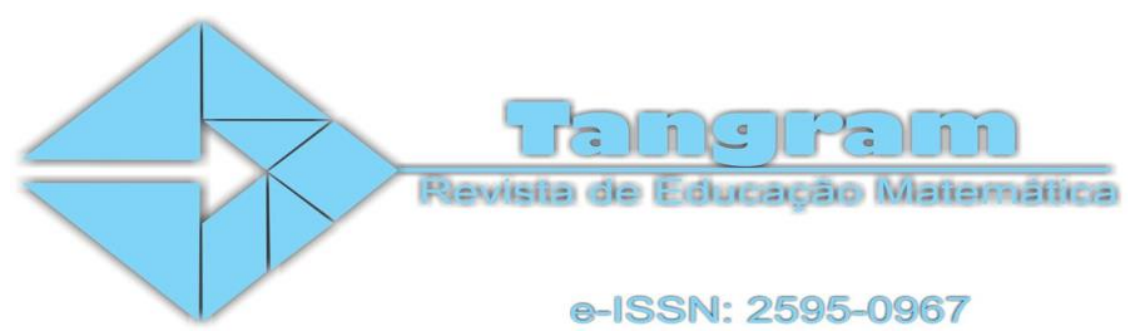

trabajar para favorecer la autonomía mental del alumnado, potenciando las estrategias creativas de resolución de problemas, la elaboración de hipótesis, la argumentación, el contraste, la negociación de significados, la construcción conjunta de soluciones y la búsqueda de formas para comunicar y representar planteamientos y resultados. En definitiva, pues, se trata de ayudar a gestionar el conocimiento, las habilidades y las emociones para conseguir un objetivo a menudo más cercano a situaciones funcionales y en contextos de vida cotidiana que a su uso académico.

Considerando esta visión del conocimiento matemático, Alsina (2016) señala que, al diseñar una práctica de enseñanza, es necesario identificar los contenidos matemáticos que pueden trabajarse en el contexto de aprendizaje, y establecer a través de qué procesos van a trabajarse. En la figura 5 se muestra la relación cartesiana entre los contenidos y los procesos matemáticos, a la vez que se ofrecen algunas orientaciones en forma de preguntas para promover una enseñanza de las matemáticas a través de los procesos.

\begin{tabular}{|c|c|c|c|c|c|}
\hline & $\begin{array}{l}\text { Resolución } \\
\text { de problemas }\end{array}$ & $\begin{array}{l}\text { Razonamien } \\
\text { to y prueba }\end{array}$ & $\begin{array}{l}\text { Comunicaci } \\
\text { ón }\end{array}$ & Conexiones & $\begin{array}{l}\text { Representac } \\
\text { ión }\end{array}$ \\
\hline $\begin{array}{l}\text { Números y } \\
\text { operacione } \\
\mathrm{s}\end{array}$ & \multirow{5}{*}{$\begin{array}{l}\text { ¿Qué } \\
\text { problema/ret } \\
\text { o voy a } \\
\text { plantear al } \\
\text { alumnado? } \\
\text { ¿Cuál es la } \\
\text { incógnita/cuấ } \\
\text { les son los } \\
\text { datos? } \\
\text { ¿Conoces } \\
\text { algún otro } \\
\text { problema }\end{array}$} & \multirow{5}{*}{$\begin{array}{l}\text { ¿Qué } \\
\text { buenas } \\
\text { preguntas } \\
\text { voy a } \\
\text { formular } \\
\text { para que el } \\
\text { àlumnado } \\
\text { argumente } \\
\text { sus ideas } \\
\text { matemáticas } \\
\text { y sus } \\
\text { acciones? }\end{array}$} & \multirow{5}{*}{$\begin{array}{l}\text { ¿Cómo voy } \\
\text { a fomentar } \\
\text { la } \\
\text { interacción? } \\
\text { (en parejas, } \\
\text { en pequeño } \\
\text { grupo, etc.) } \\
\text { ¿Qué } \\
\text { evocegectitario } \\
\text { deben } \\
\text { aprender? }\end{array}$} & \multirow{5}{*}{$\begin{array}{l}\text { cQué } \\
\text { contenidos } \\
\text { matemático } \\
\text { s se pueden } \\
\text { relạcionar? } \\
\text { bi Desde qué } \\
\text { asignatura } \\
\text { voy a } \\
\text { plantear el } \\
\text { reto? }\end{array}$} & \multirow{5}{*}{$\begin{array}{l}\text { ¿Qué tipo de } \\
\text { representaci } \\
\text { ón debe } \\
\text { hacer el } \\
\text { alumnado? } \\
\text { Verbal, } \\
\text { gráfica, } \\
\text { simbólica ... }\end{array}$} \\
\hline Álgebra & & & & & \\
\hline Geometría & & & & & \\
\hline Medida & & & & & \\
\hline $\begin{array}{l}\text { Estadística } \\
\text { y } \\
\text { probabilid } \\
\text { ad }\end{array}$ & & & & & \\
\hline
\end{tabular}

Tangram - Revista de Educação Matemática, Dourados - MS - v.3 n.2, pp. 127-159 (2020) 


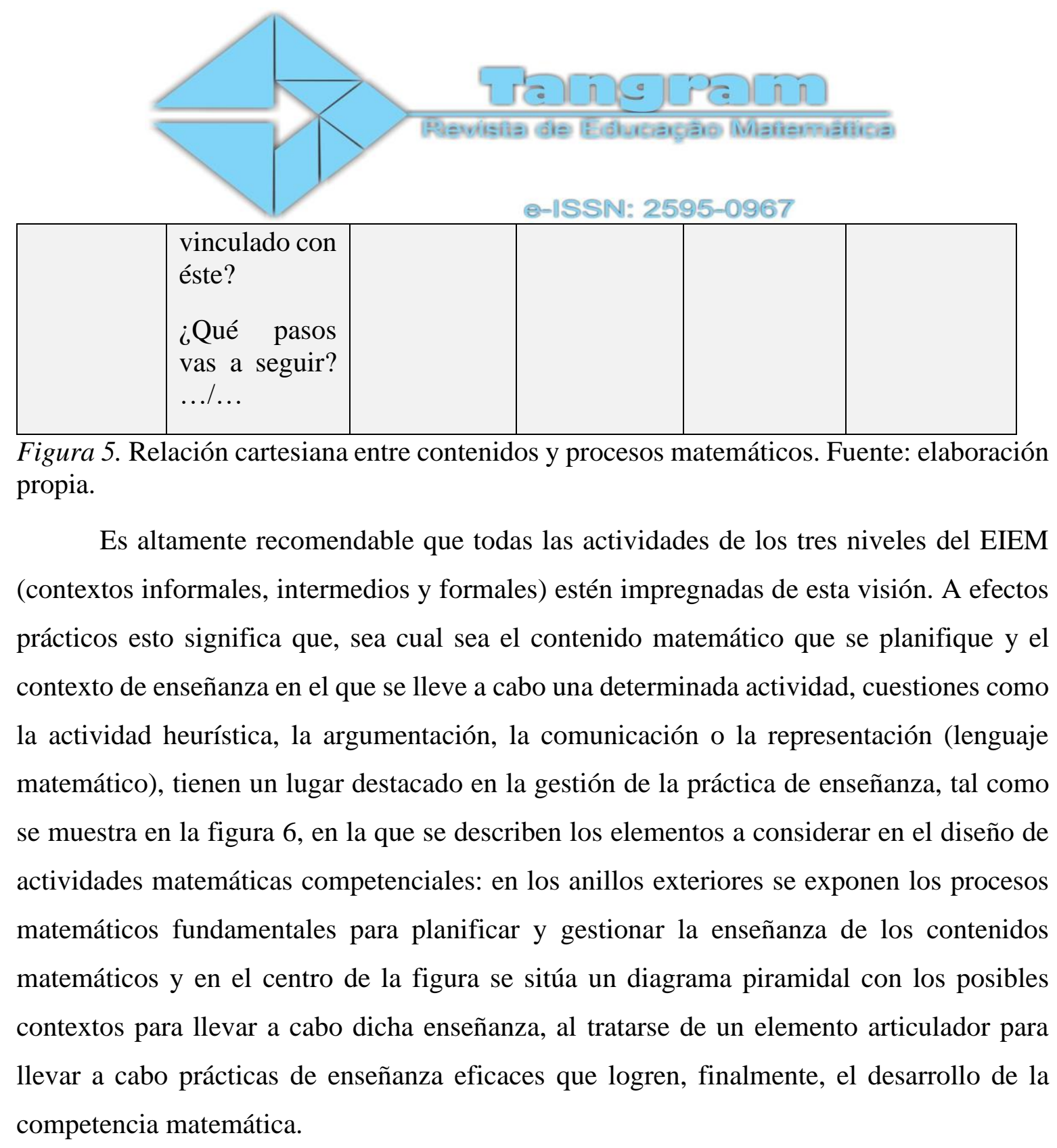

Tangram - Revista de Educação Matemática, Dourados - MS - v.3 n.2, pp. 127-159 (2020)

Este obra está licenciada com uma Licença Creative Commons Atribuição-NãoComercial-CompartilhaIgual 3.0 Brasil. 


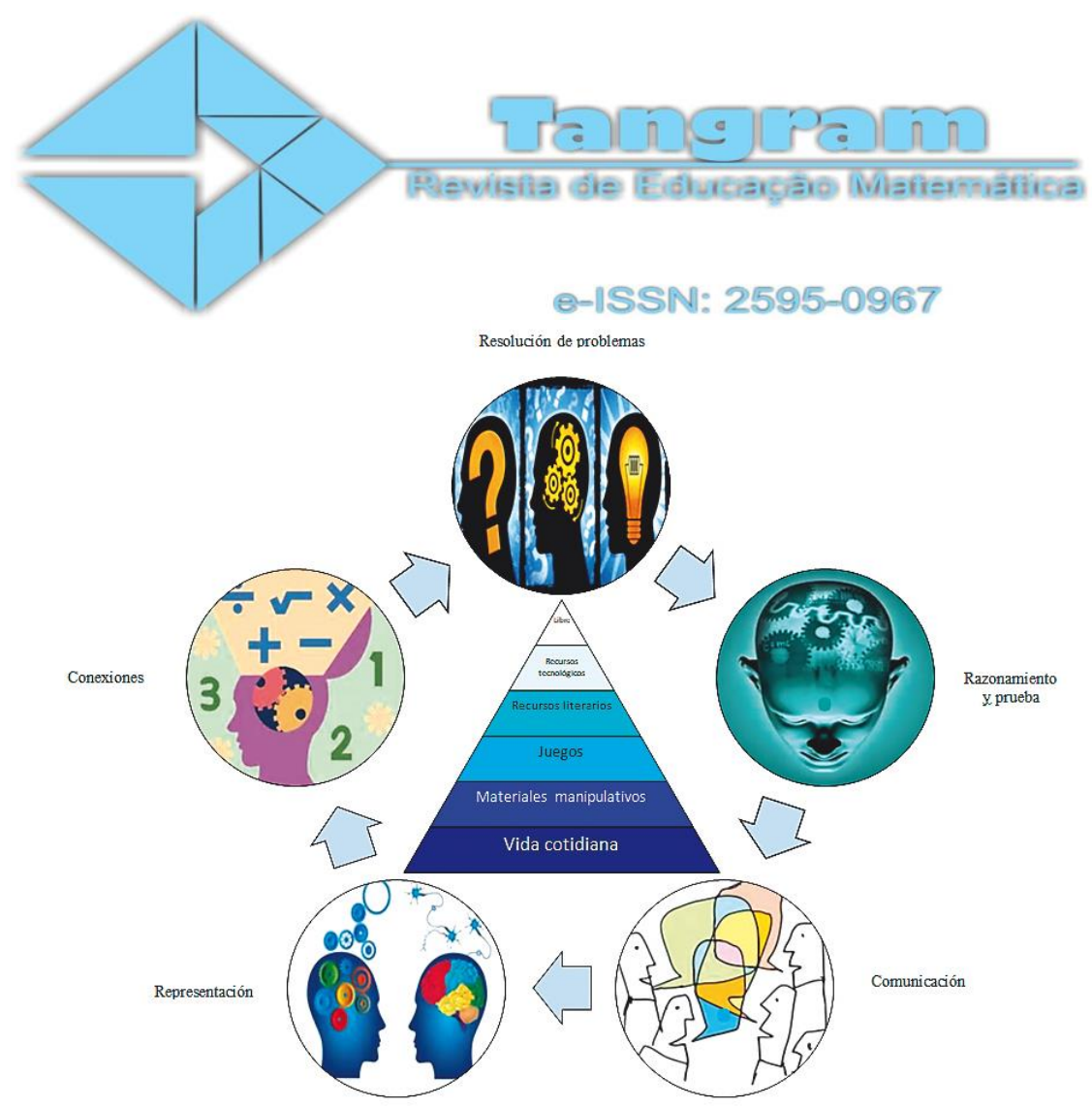

Figura 6. Elementos clave para una enseñanza competencial de las matemáticas. Fuente:

$$
\text { Alsina (2019b) }
$$

Este planteamiento no implica, sin embargo, que en la planificación y gestión de una actividad deban considerarse todos los procesos matemáticos explícitamente y de manera conjunta. Si bien la resolución de problemas es el eje articulador de todas las actividades (Ministry of Education Singapore, 2012), una determinada actividad se puede focalizar en la argumentación a través del planteamiento de buenas preguntas, en otra puede interesar promover la representación, etc. De todas formas, desde un punto de vista competencial, cuantos más procesos matemáticos pueden integrarse en una actividad, más rica es desde un punto de vista competencial.

\section{Recomendación 2. Promover prácticas de enseñanza-aprendizaje que consideren tanto al alumnado como al profesorado.}

Uno de los fundamentos del EIEM es la Teoría Sociocultural del Aprendizaje Humano de Vygostky (1978) que, como se ha indicado, considera que la personas construyen su conocimiento en un contexto social, a partir de la interacción, la negociación

Tangram - Revista de Educação Matemática, Dourados - MS - v.3 n.2, pp. 127-159 (2020) 


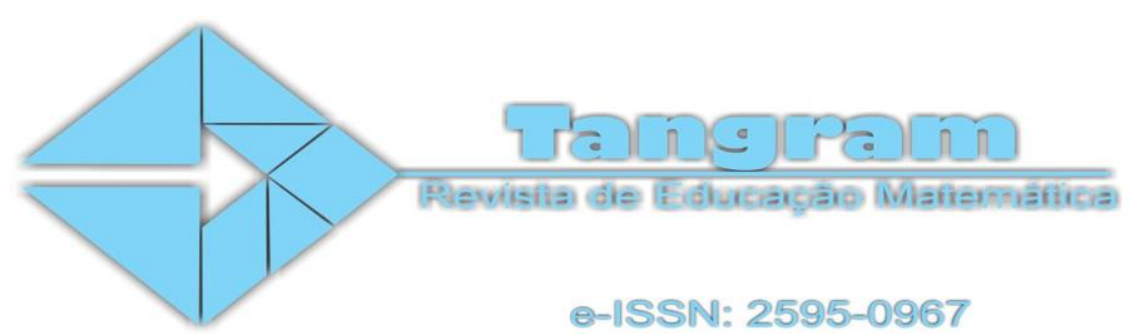

y el diálogo. Esta teoría, pues, puede ubicarse dentro del grupo de teorías denominadas Inquiry-Based Education, que basan su enfoque en el aprendizaje por indagación del alumnado, con un apoyo subsidiario del profesorado (Artigue y Blomhøj, 2013), que hace de mediador en el proceso de enseñanza-aprendizaje.

Esta visión de la enseñanza es la que predomina en la actualidad en los currículos de matemáticas, puesto que se apoyan en marcos teóricos de tipo constructivista o socioconstructivista (Godino, Batanero, Cañadas y Contreras, 2015). Así, por ejemplo, el NCTM (2003, p. 22), indica que:

Los estudiantes aprenden más y mejor cuando ellos mismos toman el control de sus aprendizajes definiendo sus objetivos y controlando su progreso. Cuando son desafiados con tareas elegidas de manera apropiada, los estudiantes adquieren confianza en su habilidad para abordar problemas difíciles, desean resolver las cosas por sí mismos, muestran flexibilidad al explorar ideas matemáticas e intentar vías de solución alternativas, y disposición para perseverar.

Esta visión puede malinterpretarse en las prácticas de enseñanza al entender que el alumnado "aprende solo", sin tener en cuenta el importante papel que tiene el profesorado. En este sentido, por ejemplo, en un estudio realizado por López y Alsina (2015) se puso de manifiesto un bajo rendimiento de un grupo de alumnos de Educación Infantil que habían aprendido matemáticas a partir de un enfoque basado principalmente en la manipulación de materiales. Al analizar las razones de este resultado, en principio contradictorio, se detectó que el papel de la maestra en la práctica de enseñanza era prácticamente nulo: proporcionaba el material, dejaba que los niños manipularan sin ninguna mediación por su parte y, una vez finalizaba la sesión, lo retiraba.

Para evitar este tipo de prácticas, en la primera recomendación ya se ha insistido en el importante rol que tiene el profesorado. Si bien es cierto que, por un lado, la enseñanza de las matemáticas a partir de los procesos matemáticos pone el foco en que el alumnado resuelva problemas, razone, argumente, comunique, conecte o represente las ideas

Tangram - Revista de Educação Matemática, Dourados - MS - v.3 n.2, pp. 127-159 (2020) 


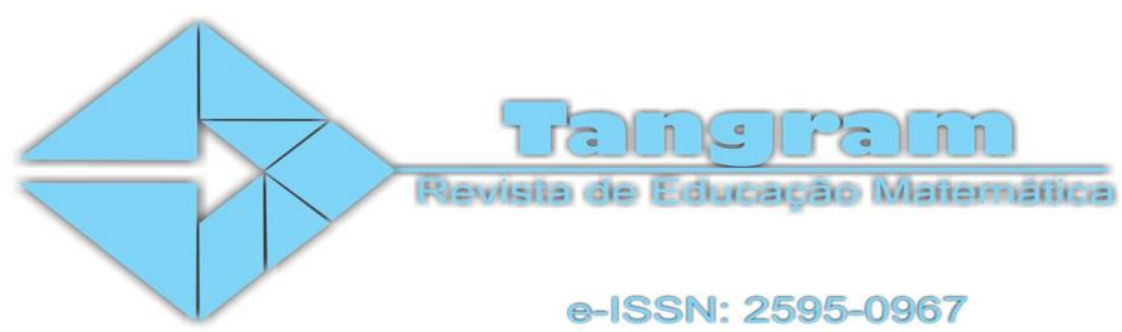

matemáticas, estas acciones no se manifiestan si el profesorado no las promueve a través, por ejemplo, de enseñar un determinado contenido matemático utilizando diversas herramientas, explicar distintas estrategias para resolver un problema, plantear preguntas efectivas previamente pensadas que inviten a argumentar, confrontar diferentes maneras de representar la solución de un problema, etc.

De ello se desprende que, como indican Godino et al. (2015), en la gestión de las prácticas de enseñanza es necesario considerar el debate entre los modelos centrados en la transmisión de conocimientos y los modelos centrados en la construcción de conocimientos: los primeros sostienen que la eficacia del proceso de estudio está ligada más a la acción docente que al descubrimiento del alumnado y, en consecuencia, focalizan su trabajo en el modelo instruccional directo y transmisivo (Mayer, 2004; Boghossian, 2006), y los segundos basan su enfoque en el aprendizaje por indagación del alumnado, con un apoyo subsidiario del profesorado (Artigue y Blomhøj, 2013).

El EIEM recomienda la combinación de ambos modelos en la práctica docente, en la línea de Godino et al. (2015) y Godino y Burgos (2020). Este planteamiento, recogiendo la primera recomendación descrita, implica una gestión de las prácticas de enseñanza que considere la enseñanza de los contenidos matemáticos a través de los procesos a partir de actividades competenciales ricas, en las que tanto el alumnado como el profesorado tengan un papel relevante: el alumnado construyendo su conocimiento y el profesorado proporcionándolo.

En un ejercicio de ingeniería didáctica, esto significa que, como ya se ha indicado reiteradamente, el profesorado debe conocer diversas maneras de actuar y tener criterios para saber cuándo, qué y por qué algo es conveniente y reflexionar sobre ello sistemáticamente, de acuerdo con el segundo fundamento del EIEM (Korthagen, 2001). Desde este prisma, y en el marco de las ideas expuestas en las figuras 5 y 6 , el profesorado es el responsable de tomar decisiones acertadas durante la práctica docente acerca de cuándo es necesario

Tangram - Revista de Educação Matemática, Dourados - MS - v.3 n.2, pp. 127-159 (2020)

Este obra está licenciada com uma Licença Creative Commons Atribuição-NãoComercial-CompartilhaIgual 3.0 Brasil. 


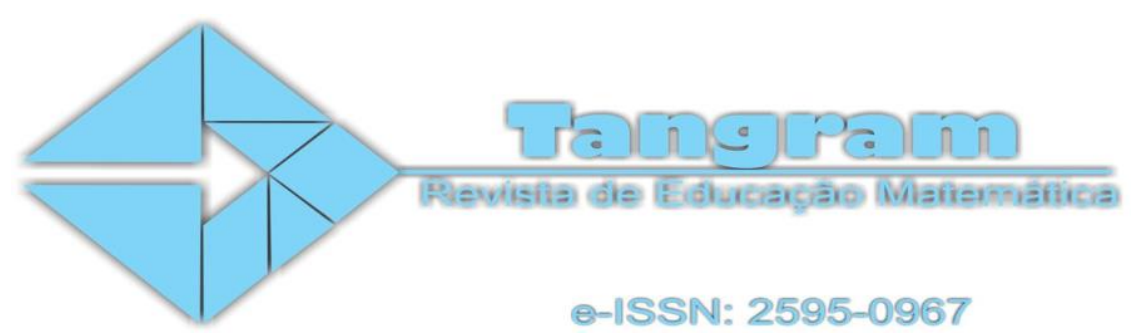

explicar un conocimiento matemático y cuándo es imprescindible que el alumnado indague y construya su conocimiento. En el EIEM se considera que, si no hay una gestión a veces indagativa y otras veces directa, el aprendizaje eficaz no es posible.

\section{Recomendación 3. Considerar contextos reales, intermedios y formales en todas las secuencias, con distinto protagonismo en función del nivel escolar.}

Esta tercera recomendación ya se empezó a proponer en el diagrama piramidal de la figura 3, en la que se muestran diversos contextos para desarrollar el pensamiento matemático y se comunica su frecuencia de uso, de más a menos, en función de la posición que ocupa cada contexto desde la base hacia la cúspide, como se ha indicado. En el marco tanto de la formación inicial como permanente, futuros docentes y profesorado en activo que han tenido acceso a la Pirámide de la Educación Matemática (Alsina, 2010) han formulado diversos interrogantes como por ejemplo ¿siempre debe darse mayor protagonismo a los contextos de la base del diagrama piramidal, independientemente del nivel escolar del alumnado?; ¿es necesario utilizar siempre todos los contextos de manera lineal, de más o menos frecuencia, para garantizar la comprensión?; o, incluso se ha polemizado alrededor de la ubicación de algún contexto, en especial los recursos tecnológicos, al considerar que están poco considerados en la secuencia de enseñanza propuesta, por su proximidad a la cúspide. En esta recomendación y la siguiente se procuran aclarar estas dudas a inquietudes, en el marco de la transposición de la Pirámide de la Educación Matemática al EIEM.

Sobre el protagonismo de cada contexto en un itinerario de enseñanza, existe cierta tendencia entre el profesorado a considerar que los contextos informales (situaciones cotidianas, materiales manipulativos y juegos) son exclusivos de las primeras edades. En el EIEM se quiere romper rotundamente con esta visión y, en su lugar, se asume la relevancia de estos contextos para el aprendizaje de las matemáticas con comprensión en todas las edades.

Tangram - Revista de Educação Matemática, Dourados - MS - v.3 n.2, pp. 127-159 (2020)

Este obra está licenciada com uma Licença Creative Commons Atribuição-NãoComercial-CompartilhaIgual 3.0 Brasil. 


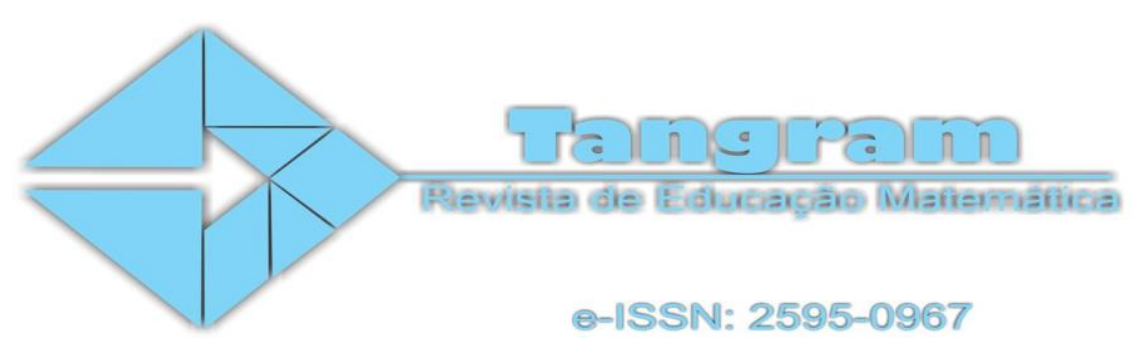

Sobre las situaciones cotidianas, por ejemplo, Reeuwijk (1997) señala que ayudan al alumnado a comprender por qué las matemáticas son útiles y necesarias; contribuyen a que el alumnado entienda cómo se emplean las matemáticas en la vida cotidiana; favorecen que los propios alumnos y alumnas aprendan a usar las matemáticas en la sociedad; incrementan el interés por las matemáticas; despiertan la creatividad del alumnado, al impulsarlos a utilizar estrategias informales y de sentido común cuando afrontan, por ejemplo, la resolución de un problema o de un juego; y, finalmente, pueden actuar como mediadores entre la situación concreta y las matemáticas abstractas.

En relación a los materiales manipulativos, Alsina y Planas (2008, p. 50) indican que:

La manipulación es mucho más que una manera divertida de desarrollar aprendizajes. La manipulación de materiales es en ella misma una manera de aprender que debe hacer más eficaz el proceso de aprendizaje, sin hacerlo necesariamente más rápido. Por otra parte, el uso de materiales es una manera de promover la autonomía del aprendiz ya que se limita la participación de los otros, principalmente del adulto, en momentos cruciales del proceso de aprendizaje.

Y respecto a los juegos, algunas de las principales ventajas de su uso para aprender matemáticas son las siguientes (Alsina, 2004): son la parte de la vida más real del alumnado, por lo que usándolos como recurso metodológico, se traslada la realidad del alumnado a la escuela y permiten descubrir la necesidad y la utilidad de aprender matemáticas; son enormemente motivadores; tratan distintos tipos de conocimientos, habilidades y actitudes hacia las matemáticas; permiten afrontar contenidos matemáticos nuevos sin miedo al fracaso inicial; e impulsan el aprendizaje a partir del propio error y del error de los demás.

Desde este prisma, es obvio que los contextos informales son imprescindibles para garantizar la comprensión de las ideas matemáticas en todos los niveles escolares. El posicionamiento del EIEM es, pues, claro en este sentido; sin embargo, sí que se considera que el protagonismo de estos contextos es flexible, en función de la edad del alumnado. Esta idea, llevada a la práctica docente, significa que, mientras que en las primeras edades debe

Tangram - Revista de Educação Matemática, Dourados - MS - v.3 n.2, pp. 127-159 (2020) 


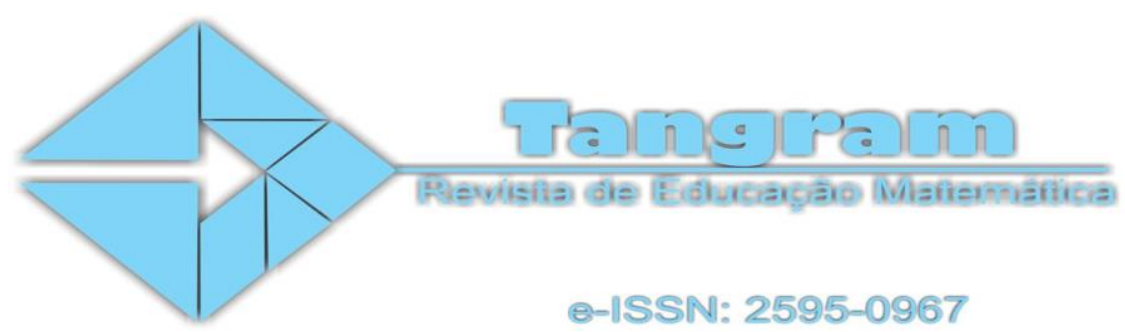

haber una fuerte presencia de los contextos informales (situaciones reales, materiales manipulativos y juegos) para permitir visualizar las ideas matemáticas de manera concreta, en los últimos cursos debe ir reduciéndose esta presencia para dar mayor protagonismo a los contextos intermedios y formales (recursos tecnológicos y gráficos, principalmente) para lograr la formalización y la institucionalización de los aprendizajes, pero sin olvidar que los contextos informales son imprescindibles para enseñar nuevas ideas matemáticas en cualquier edad. Esto es importante si se quiere dar respuesta a las necesidades reales del alumnado para aprender matemáticas, puesto que existe otra tendencia entre una parte del profesorado, que es falsa, y que consiste en pensar que el alumnado de más edad debe pasar directamente a lo formal o abstracto, sin espacio para lo situacional o concreto, provocando en consecuencia aprendizajes mecánicos y ausentes de comprensión.

\section{Recomendación 4. Garantizar el principio de abstracción progresiva, desde lo concreto hacia lo abstracto.}

Todavía en el marco de la planificación de un itinerario de enseñanza, y con el propósito de dar respuesta a otros interrogantes alrededor de los distintos contextos y su uso, es necesario precisar que no es imprescindible utilizar siempre todos los contextos incluidos en los tres niveles del EIEM de manera lineal, de más o menos frecuencia, para garantizar la comprensión.

En la planificación de una secuencia de enseñanza de un determinado contenido se puede, por ejemplo, plantear un problema real, promover el uso de un recurso tecnológico como apoyo y un contexto más formal para avanzar hacia la formalización; o bien se puede plantear un reto a partir de un material manipulativo, seguir con un recurso literario y finalizar con un recurso gráfico, por citar algunas combinacions posibles. En definitiva, se trata de tener presente que, a través de los diversos contextos que componen un itinerario de enseñanza de las matemáticas y las actividades que se plantean en cada contexto, debe garantizarse primero la visualización de las ideas matemáticas de manera concreta (lo que

Tangram - Revista de Educação Matemática, Dourados - MS - v.3 n.2, pp. 127-159 (2020)

Este obra está licenciada com uma Licença Creative Commons Atribuição-NãoComercial-CompartilhaIgual 3.0 Brasil. 


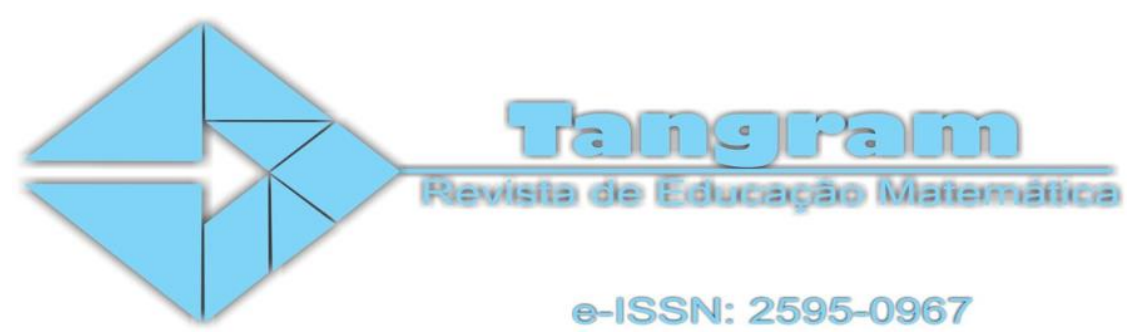

en el argot del profesorado se denomina a menudo vivenciación) mediante situaciones de vida cotidiana, materiales manipulativos o juegos; a continuación ofrecer apoyos para avanzar hacia la esquematización y generalización progresiva, usando recursos literarios o bien tecnológicos, principalmente; y terminar la secuencia con recursos que promuevan la representación con notaciones convencionales, es decir, la simbolización y la abstracción, para asegurar así la formalización del conocimiento matemático, de acuerdo con las posibilidades del alumnado.

Finalmente, debe hacerse un especial hincapié en la ubicación de los recursos tecnólogicos en un itinerario de enseñanza, por el enorme protagonismo que han adquirido estos recursos en los últimos años debido a la avalancha de Applets, en muchos casos de acceso gratuito, o la consideración de la robótica en el aula desde las primeras edades. Desde el punto de vista del EIEM, se asume que aunque estos recursos ofrecen muchas posibilidades y suelen garantizar la implicación del alumnado, no hay que olvidar que antes del entorno simulado, deben visualizarse las ideas matemáticas de manera concreta, de acuerdo con Alsina y Planas (2008), razón por la cual se considera que estos recursos promueven la esquematización y la generalización progresiva siempre que, previamente, se hayan podido visualizar las ideas matemáticas en contextos informales. Por este motivo, los recursos tecnológicos se ubican en el conjunto de contextos intermedios, que hacen de puente entre el nivel situacional y formal.

\section{Recomendación 5. Disponer de criterios objetivos para la selección de los contextos de enseñanza de las matemáticas.}

Actualmente, el profesorado en activo y los futuros docentes tienen a su alcance una cantidad impresionante de recursos para enseñar matemáticas de procedencia muy distinta, desde expertos en el campo de la educación matemática hasta empresas que buscan un espacio para hacer negocio, sin olvidar la gran cantidad de propuestas de distintos tipos que pueden encontrarse en Internet, algunas veces sin ningún filtro que garantice su calidad.

Tangram - Revista de Educação Matemática, Dourados - MS - v.3 n.2, pp. 127-159 (2020)

Este obra está licenciada com uma Licença Creative Commons Atribuição-NãoComercial-CompartilhaIgual 3.0 Brasil. 


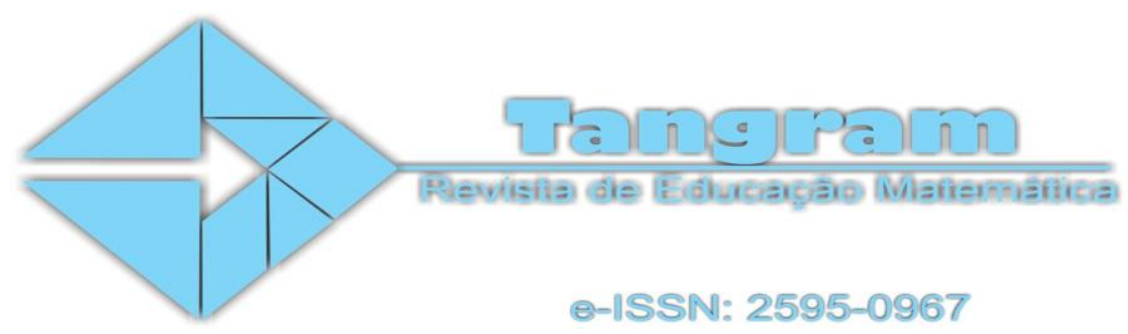

Resulta evidente que este alud de recursos requiere que los profesionales de la educación, a través de la formación inicial y permanente, dispongan de conocimientos matemáticos y didácticos adecuados para tener criterios objetivos, es decir, respaldados por la investigación, para seleccionar recursos que garanticen un buen aprendizaje de las matemáticas.

Ya se ha hecho referencia repetidamente al papel del aprendizaje realista para que el profesorado pueda disponer de criterios para saber cuándo, qué y por qué algo es conveniente y reflexionar sobre ello sistemáticamente (Korthagen, 2001). Es evidente, pues, la reflexión sistemática es imprescindible para indagar sobre interrogantes que genera la planificación de actividades, como por ejemplo ¿cómo puedo saber si un material manipulativo es adecuado para enseñar un determinado contenido matemático?, ¿del conjunto de Applets que conozco, cual es el mejor?, ¿qué libro de texto, de los que me ofrecen las editoriales, propone actividades de mejor calidad pedagógica? o ¿qué otros contextos, aparte del libro, puedo utilizar para garantizar el aprendizaje con comprensión?

Si bien es cierto que al aprendizaje realista empodera al profesorado para disponer de criterios para saber cuándo, qué y por qué algo es conveniente, desde el EIEM se considera que es necesario complementar estos criterios fundamentados en la reflexión sistemática con otras herramientas de análisis que permitan seleccionar cualitativamente los recursos que se tienen al alcance.

En este sentido, y en coherencia con la primera recomendación acerca de la aplicación del EIEM en el aula, que se centra en la planificación y gestión de la enseñanza de los contenidos a través de los procesos matemáticos, se considera que, mediante una gestión adecuada, un buen recurso debería facilitar la resolución de problemas, el razonamiento y la prueba, la comunicación, las conexiones y/o la representación. En este sentido, se propone como herramienta de análisis, el instrumento validado ETMAP (por las siglas en inglés, Evaluating the Teaching of Mathematics through Processes), que permite

Tangram - Revista de Educação Matemática, Dourados - MS - v.3 n.2, pp. 127-159 (2020) 


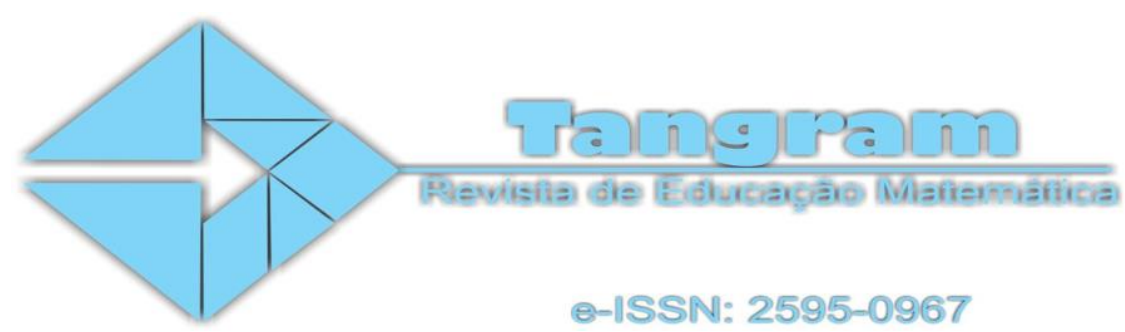

orientar al profesorado sobre qué aspectos se deben considerar para llevar a cabo una enseñanza de las matemáticas a través de los procesos matemáticos $\mathrm{y}$, a la vez, permite analizar la presencia de estos procesos en sus prácticas de enseñanza. Este instrumento aporta siete indicadores para cada proceso, y puede consultarse íntegramente en Alsina, Maurandi, Ferre y Coronata (2020).

La educación matemática, en el marco de distintos modelos teóricos, ofrece otras herramientas que permiten realizar análisis objetivos. En este sentido, destacamos por ejemplo los criterios de idoneidad didáctica propuestos por el Enfoque Ontosemiótico (EOS) del conocimiento y la instrucción matemáticos: idoneidad epistémica, cognitiva, interaccional, mediacional, emocional, y ecológica, que pueden consultarse íntegramente en Godino, Batanero, y Font (2007). Cabe señalar que la idoneidad mediacional, en concreto, permite valorar la adecuación de los recursos materiales y temporales utilizados en el proceso de instrucción.

\section{Consideraciones finales}

En este artículo se ha fundamentado y descrito el Enfoque de los Itinerarios de Enseñanza de las Matemáticas (EIEM) y se han proporcionado cinco recomendaciones iniciales para su aplicación en el aula. Como se ha indicado en la introducción, el EIEM pretende ser una aportación modesta, pero útil y aplicable al aula, para todo el profesorado de matemáticas que manifiesta el deseo de mejorar sus prácticas de enseñanza, pero que a menudo no sabe cómo hacerlo. El EIEM no pretende, por supuesto, ser la solución de todos los problemas ni dar respuesta a todas las lagunas detectadas por la investigación en educación matemática en relación a la práctica del profesor, pero sí proporcionar algunas orientaciones que ayuden a su desarrollo profesional con el propósito de mejorar dicha práctica y promover un impacto positivo en el aprendizaje matemático del alumnado.

Tangram - Revista de Educação Matemática, Dourados - MS - v.3 n.2, pp. 127-159 (2020) 


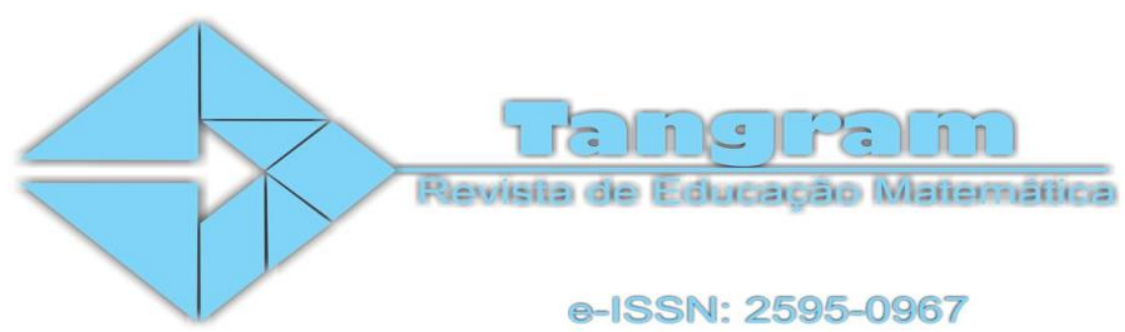

Muy sintéticamente, como se ha indicado, el EIEM se sustenta principalmente en tres pilares engranados entre ellos: la Perspectiva Sociocultural del Aprendizaje Humano (Vygostsky, 1978); el Modelo Realista de Formación del Profesorado (Melief, Tigchelaar y Korthagen, 2010; Tigchelaar, Melief, Van Rijswijk y Korthagen, 2010), adaptado a la formación del profesorado de matemáticas (Alsina, 2019c); y la Educación Matemática Realista (EMR) de Freudenthal (1991), junto con la consideración de otras aportaciones de organismos y autores como el NCTM (2003) o Godino et al. (2015), entre otros. En este sentido, algunas de las ideas principales que impregnan las prácticas de enseñanza de las matemáticas basadas en el EIEM son las siguientes: el aprendizaje se produce en un contexto social y cultural, a partir de la interacción, la negociación y el diálogo (Vygotsky, 1978); el profesorado debe conocer muchas maneras de actuar y ejercitarlas en la práctica, junto con disponer de criterios para saber cuándo, qué y por qué algo es conveniente y reflexionar sobre ello sistemáticamente (Korthagen, 2001); y, finalmente, la idea de que la matemática es una actividad humana que surge como matematización (organización) de la realidad, y su comprensión de desarrolla a partir de distintos niveles, desde el situacional (en el contexto de la situación) al formal (procedimientos estándares y notaciones convencionales), considerando además otros principios como la reinvención guiada, la interacción e la interconexión (Freudenthal, 1991).

Con el propósito de ofrecer algunos andamios al profesorado, se han ofrecido cinco recomendaciones iniciales para aplicar el EIEM en el aula: 1) planificar y gestionar la enseñanza de los contenidos a través de los procesos matemáticos, es decir, promover una enseñanza que implique pensar y hacer, más que memorizar definiciones y procedimientos (Alsina et al., 2020); 2) promover prácticas de enseñanza-aprendizaje que consideren tanto al alumnado como al profesorado, en las que haya espacio tanto para que el alumnado indague y construya su conocimiento como para que el profesorado explique de forma directa un conocimiento matemático (Godino et al., 2015; Godino y Burgos, 2020); 3) considerar contextos reales, intermedios y formales, con distinto protagonismo en función

Tangram - Revista de Educação Matemática, Dourados - MS - v.3 n.2, pp. 127-159 (2020) 


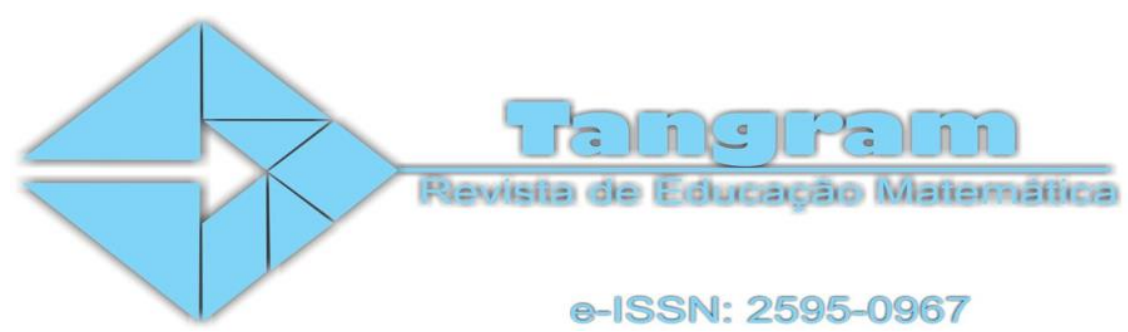

del nivel escolar, es decir, que se considere que si bien es cierto que en los primeros niveles los contextos informales tienen un especial protagonismo y que se debe ir disminuyendo su presencia a medida que se avanza la escolaridad, no hay que olvidar que son imprescindibles en todas las edades para asegurar la comprensión; 4) garantizar el principio de abstracción progresiva, desde lo concreto hacia lo abstracto, de manera que a lo largo de un itinerario se considere la visualización, la manipulación, la simbolización y la abstracción; y 5) disponer de criterios objetivos para la selección de los contextos de enseñanza de las matemáticas, a partir de distintas herramientas (Alsina et al., 2020; Godino et al., 2007).

Desde la perspectiva de la innovación educativa, en futuros trabajos será necesario diseñar secuencias de enseñanza fundamentadas en el EIEM para promover, desde la perspectiva del desarrollo profesional del profesorado de matemáticas, mejoras en la enseñanza de los distintos contenidos: numeración y cálculo, álgebra temprana, geometría, medida, y estadística y probabilidad. Y desde la perspectiva de la investigación en educación matemática, será necesario diseñar estudios que analicen el efecto del EIEM desde una doble perspectiva: a) desde la perspectiva del alumnado, para analizar las actitudes hacia los distintos contextos de enseñanza (interés, motivación, etc.), junto con analizar el rendimiento en función del tipo de contexto; y b) desde la perspectiva del profesorado, para analizar las actitudes/creencias sobre la enseñanza de los contenidos matemáticos en los diferentes contextos, entre otros aspectos. Finalmente, será necesario también implementar actividades formativas sobre el EIEM, tanto en la formación inicial como permanente, que estén diseñadas a partir de modelos formativos transformacionales, como por ejemplo el Modelo Transformacional de la Competencia Profesional del Profesorado de Matemáticas (Alsina y Mulà, 2019), y analizar su efecto para transformar la práctica del profesorado de matemáticas, en el marco de una escuela que se renueva.

Tangram - Revista de Educação Matemática, Dourados - MS - v.3 n.2, pp. 127-159 (2020)

Este obra está licenciada com uma Licença Creative Commons Atribuição-NãoComercial-CompartilhaIgual 3.0 Brasil. 


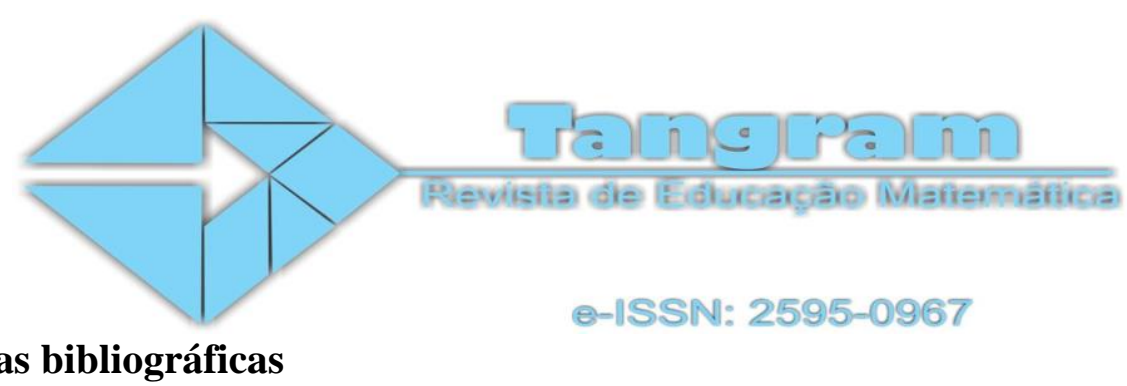

\section{Referencias bibliográficas}

Alsina, Á. (2004). Desarrollo de competencias matemáticas con recursos lúdicomanipulativos para niños y niñas de 6 a 12 años. Madrid: Narcea S.A. de Ediciones.

Alsina, Á. (2010). La "pirámide de la educación matemática", una herramienta para ayudar a desarrollar la competencia matemática. Aula de Innovación Educativa, 189, $12-$ 16.

Alsina, Á. (2012). Más allá de los contenidos, los procesos matemáticos en Educación Infantil. Edma 0-6: Educación Matemática en la Infancia, 1(1), 1-14.

Alsina, Á. (2016). Diseño, gestión y evaluación de actividades matemáticas competenciales en el aula. Épsilon, Revista de Educación Matemática, 33(1), 7-29.

Alsina, Á. (2018). Seis lecciones de educación matemática en tiempos de cambio: itinerarios didácticos para aprender más y mejor. Padres y Maestros, 376, 13-20.

Alsina, Á. (2019b). Itinerarios didácticos para la enseñanza de las matemáticas (6-12 años). Barcelona: Editorial Graó.

Alsina, Á. (2019a). La educación matemática infantil en España: ¿qué falta por hacer? Números. Revista de Didáctica de las Matemáticas, 100, 85-108.

Alsina, Á. (2019c). Hacia una formación transformadora de futuros maestros de matemáticas: avances de investigación desde el modelo realista-reflexivo. Unipluriversidad, 19(2), 60-79.

Alsina, A. (2020). Itinerario de enseñanza para el álgebra temprana. Revista Chilena de Educación Matemática, 12(1), 5-20.

Alsina, Á. (en prensa). Cinco prácticas productivas para una enseñanza de las matemáticas a través de los procesos. Saber \& Educar.

Alsina, Á. y Mulà, I. (2019). Advancing towards a transformational professional competence model through reflective learning and sustainability: The case of mathematics teacher education. Sustainability, 11, 4039.

Alsina, Á. y Planas, N. (2008). Matemática inclusiva. Propuestas para una educación matemática accesible. Madrid: Narcea S.A. de Ediciones.

Tangram - Revista de Educação Matemática, Dourados - MS - v.3 n.2, pp. 127-159 (2020)

Este obra está licenciada com uma Licença Creative Commons Atribuição-NãoComercial-CompartilhaIgual 3.0 Brasil. 


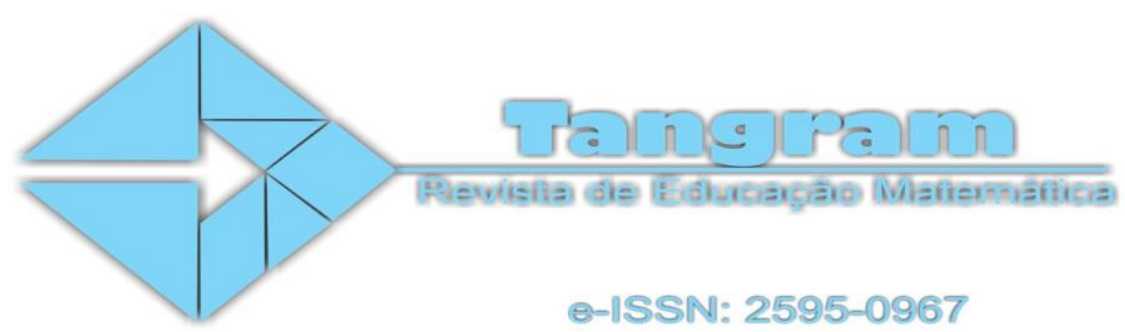

Alsina, Á., Maurandi, A., Ferre, E., y Coronata, C. (2020). Validating an Instrument to Evaluate the Teaching of Mathematics Through Processes. International Journal of Science and Mathematics Education.

Alsina, Á., y Domingo, M. (2010). Idoneidad didáctica de un protocolo sociocultural de enseñanza y aprendizaje de las matemáticas. Revista Latinoamericana de Investigación en Matemática Educativa, 13(1), 7-32.

Artigue, M., y Blomhøj, M. (2013). Conceptualizing inquiry-based education in mathematics. ZDM Mathematics Education 45, 797-810.

Badillo, E., Climent, N., Fernández, C. y González, Mª T. (2019). Investigación sobre el profesor de matemáticas: práctica de aula, conocimiento, competencia y desarrollo profesional. Salamanca: Ediciones Universidad Salamanca.

Boghossian, P. (2006). Behaviorism, constructivism, and Socratic pedagogy. Educational Philosophy and Theory, 38(6), 713-722.

Chevallard, Y. (1991). La transposición didáctica. Del saber sabio al saber enseñado. Buenos Aires: Aique.

De Corte, E., Greer, B., y Verschaffel, L. (1996): Mathematics Teaching and Learning. En D. Berliner, y C. Calfee (Eds.), Handbook of Educational Psychology (pp. 491549). Nueva York: Simon \& Schuster Macmillan.

Esteve, O. y Alsina, Á. (2010). Hacia el desarrollo de la competencia profesional del profesorado. En O. Esteve, K. Melief y Á. Alsina (Eds.), Creando mi profesión. Una propuesta para el desarrollo profesional del profesorado (pp. 7-18). Barcelona: Editorial Octaedro

Fauzan, A., Plomp, T., y Slettenhaar, D. (2002). Traditional mathematics education vs. realistic mathematics education: Hoping for Changes. En Proceedings of the 3rd International Mathematics Education and Society Conference (pp. 1-4). Copenhagen: Centre for Research in Learning Mathematics.

Freudenthal, H. (1991). Revisiting mathematics education. Dordrecht: Kluwer Academic Publishers.

Tangram - Revista de Educação Matemática, Dourados - MS - v.3 n.2, pp. 127-159 (2020) 


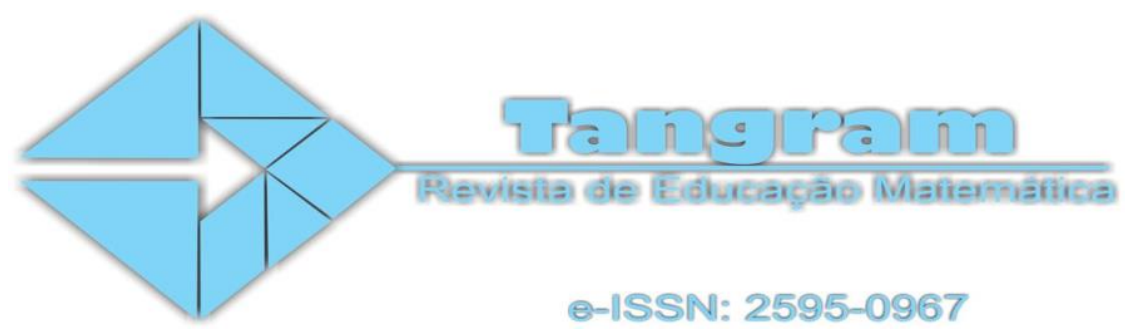

Godino, J. D. Batanero, C. y Font, V. (2007). The onto-semiotic approach to research in mathematics education. ZDM. The International Journal on Mathematics Education, 39 (1-2), 127-135.

Godino, J. D. y Burgos, M. (2020). Interweaving transmission and inquiry in mathematics and sciences instruction. En K. O. Villalba-Condori et al. (Eds.), CISETC 2019, CCIS 1191 (pp. 6-21). Springer Nature Switzerland AG.

Godino, J. D., Batanero, C., Cañadas, G. R. y Contreras, J. M. (2015). Articulación de la indagación y transmisión de conocimientos en la enseñanza y aprendizaje de las matemáticas. En B. D’Amore y M. I. Fandiño (Eds.), Congreso Internacional Didáctica de la Matemática. Una mirada internacional empírica y teórica (pp. 249269). Bogotá: Universidad de la Sabana.

Heuvel-Panhuizen, M. (2002). Realistic mathematics education as work in progress. En F. L. Lin (Ed.), Common sense in mathematics education. Proceedings of 2001 The Netherlands and Taiwan Conference on Mathematics Education (pp. 1-43). Taiwan: National Taiwan Normal University.

Ivic, I. (1994). Lev Semionovick Vygotsky (1896-1934). Perspectivas: Revista Internacional de Educación Comparada, 34 (3-4), 773-799.

Korthagen, F. A. (2001). Linking practice and theory. The pedagogy of realistic teacher education. Londres: Lawrence Erlbaum Associates.

Leóntiev, A.N. (1981). Problemas del desarrollo de la psiquis. Moscú: Universidad de Moscú.

Lerman, S. (2000). The social turn in mathematics education research. En J. Boaler (Ed.), Multiple perspectives on mathematics teaching and learning (pp. 19-44), Westport, CT: Ablex.

Lerman, S. (2001). Cultural, Discursive Psychology: A Sociocultural Approach to Studying the Teaching and Learning of Mathematics. Educational Studies in Mathematics, $46(1), 87-113$.

López, M. y Alsina, A. (2015). La influencia del método de enseñanza en la adquisición de conocimientos matemáticos en Educación Infantil. Edma 0-6, Educación Matemática en la Infancia, 4(1), 1-10.

Tangram - Revista de Educação Matemática, Dourados - MS - v.3 n.2, pp. 127-159 (2020) 


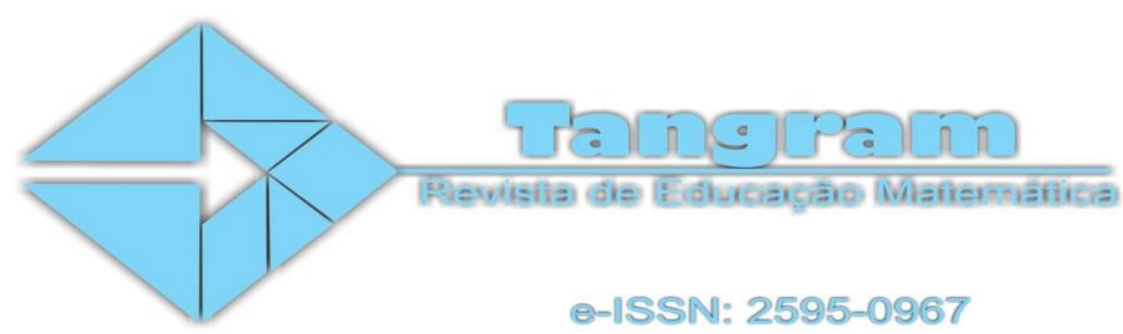

Mayer, R. E. (2004). Should there be a three-strikes rule against pure discovery learning? American Psychologist, 59 (1), 14-19.

Melief, K., Tigchelaar, A., y Korthagen, K. (2010). Aprender de la práctica. En O. Esteve, K. Melief, y Á. Alsina (Eds.), Creando mi profesión. Una propuesta para el desarrollo profesional del profesorado (pp. 19-38). Barcelona: Octaedro.

Ministry of Education Singapore (2012). Mathematics Syllabus. Primary One to Six. Singapore: Curriculum Planning and Development Division.

National Comission on Teaching and America's Future (1996). Whats Matters Most: Teaching for America's Future. Nueva York: National Comission on Teaching and America's Future.

NCTM (2003). Principios y estándares para la educación matemática. Sevilla: SAEM Thales.

Radford, L. (2006). Elementos de una teoría cultural de la objetivacion. Relime (Número esp.), 103-129.

Reeuvijk, M.V. (1997). Las matemáticas en la vida cotidiana y la vida cotidiana en las matemáticas. UNO, Revista de Didáctica de las Matemáticas, 12, 9-16.

Remillard, J. T. (2000). Can curriculum materials support teachers' learning? Elementary School Journal, 100(4), 331-350.

Schmittau, J. (2003). Cultural-Historical Theory and Mathematics Education. A Vygotsky's Educational Theory in Cultural Context. Cambridge: Cambridge University Press.

Stylianides, G. J. (2009). Reasoning-and-proving in school mathematics textbooks. Mathematical thinking and learning, 11(4), 258-288.

Tigchelaar, A., Melief, K., Van Rijswijk, M., y Korthagen, K. (2010). Elementos de una posible estructura del aprendizaje realista en la formación inicial y permanente del profesorado. En O. Esteve, K. Melief, y Á. Alsina (Eds.), Creando mi profesión. Una propuesta para el desarrollo profesional del profesorado (pp. 39-64). Barcelona: Octaedro.

Tangram - Revista de Educação Matemática, Dourados - MS - v.3 n.2, pp. 127-159 (2020) 


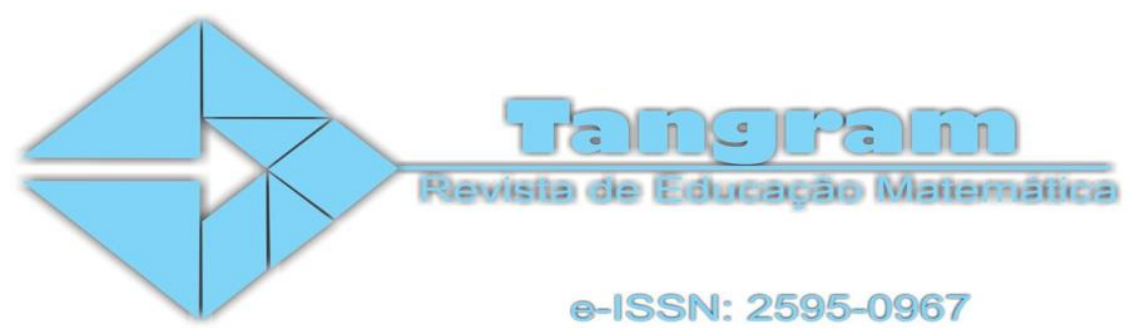

Treffers, A. (1987). Three Dimensions. A Model of Goal and Theory Description in Mathematics Instruction - The Wiskobas Project. Dordrecht: Reidel Publishing Company.

Vygotsky, L.S. (1978). Mind in society. The development of higher psychological processes. Cambridge (Mass): Harward University Press.

Wertsch, J. V. (1985). Vygotsky y la formación social de la mente. Barcelona: Paidós.

Wertsch, J. V. (1991). Voces de la mente. Un enfoque sociocultural para el estudio de la acción mediada. Madrid: Aprendizaje Visor.

Wertsch, J.V. (2013). Vygotsky y la formación social de la mente. México: Paidós.

\section{Contribuições dos Autores}

$1^{\text {a }}$ autor: conceitualização; curadoria de dados; análise formal; investigação; metodologia; visualização; redação - rascunho original; redação - revisão e edição.

Tangram - Revista de Educação Matemática, Dourados - MS - v.3 n.2, pp. 127-159 (2020) 